\title{
Variáveis contextuais como moderadoras da relação grh-desempenho organizacional: uma metanálise
}

\author{
Contextual variables as moderators of the relationship \\ hrm-organizational performance: a meta-analysis
}

\author{
Agostinha Mafalda Barra de Oliveira ${ }^{1}$ \\ Luis Fernández González ${ }^{2}$ \\ Alexandre José de Oliveira ${ }^{3}$
}

\begin{abstract}
Resumo: Nas últimas décadas, muitos estudos têm sido realizados a fim de verificar a relação entre gestão de recursos humanos (GRH) e desempenho organizacional. Revisores qualitativos e quantitativos dos resultados destes estudos concluíram que há uma relação positiva entre GRH e desempenho organizacional. No entanto, com base no alto índice de heterogeneidade apresentado nos resultados destes estudos, estes revisores apontaram para a interferência de variáveis contextuais nesta relação. Por este motivo, a fim de contribuir com o entendimento desta relação, testamos três modelos de variáveis moderadoras de tipo contextual: contexto geográfico, setor de atividade e tipos de empregados por meio de uma metanálise dos resultados de 194 registros de estudos empíricos sobre GRH e desempenho organizacional, realizados e/ou publicados entre 1985 e 2008. Das três variáveis testadas, confirmamos as variáveis: setor de atividade e contexto geográfico como moderadoras. Por outro lado, não confirmamos que a variável tipo de empregado é uma variável moderadora da relação GRH-desempenho organizacional.
\end{abstract}

Palavras-chave: Gestão de recursos humanos. Indicadores de desempenho. Métodos quantitativos.

\begin{abstract}
In the past decades, several studies have been carried out to verify the relationship between human resources management (HRM) and organizational performance. Qualitative and quantitative reviewers of the results of these studies concluded that there is a positive relationship between HRM and organizational performance. However, based on the high rates of heterogeneity reported in these studies, these reviewers point out to the interference of contextual variables in this relationship. For this reason, aiming to contribute to the understanding of this relationship, we have tested three models of contextual variable moderators: geographical context, sector of activity, and type of employees through a meta-analysis of the results of the records of 194 empirical studies on HRM and organizational performance, which were conducted and/or published from 1985 to 2008. Of the three variables tested, we confirm the variables sector of activity and geographical context as moderators, but we did not confirm the variable type of employee is a moderator of the HRM-organizational performance relationship.
\end{abstract}

Keywords: Human resources management. Organizational performance. Meta-analysis. Contextual variable.

\section{Introdução}

Nas últimas décadas, muitos estudos têm sido realizados a fim de verificar a relação existente entre práticas de Gestão de Recursos Humanos (GRH) e desempenho organizacional (ARTHUR, 1994; DELANEY; HUSELID, 1996; DELERY; DOTY, 1996; HUSELID, 1995; HUSELID; JACKSON; SCHULER, 1997; MACDUFFIE, 1995; YOUNDT et al., 1996). Um exemplo disso, no âmbito desta publicação, é o estudo de Jabbour et al. (2012). Esse estudo ofereceu uma excelente contribuição ao tema e, mais especificamente, aos Gestores de Operação e Produção, ao confirmarem a hipótese de que a GRH influencia positivamente o desempenho das operações das empresas do segmento de autopeças e componentes automotivos, no contexto brasileiro.

De forma mais ampla, revisores qualitativos dos resultados desses estudos sugeriram que há uma relação positiva entre práticas de GRH e desempenho organizacional (LACOMBE, 2004; ROGERS; WRIGHT, 1998; WOOD, 1999; WRIGHT et al., 2005)

\footnotetext{
${ }^{1}$ Departamento de Agrotecnologia e Ciências Sociais, Universidade Federal Rural do Semi-Árido - UFERSA, CEP 59625-900, Mossoró, RN, Brasil, e-mail: agostinhamafalda@ufersa.edu.br

${ }^{2}$ Faculdad de Economía y Empresa, Universidad de Salamanca - USAL, 37007, Salamanca, Espanha, e-mail: lgf@usal.es

${ }^{3}$ Departamento de Ciências Ambientais e Tecnológicas, Universidade Federal Rural do Semi-Árido - UFERSA, CEP 59625-900, Mossoró, RN, Brasil, e-mail: alexandreoliveira@ufersa.edu.br
} 
ou, mais especificamente, entre práticas de trabalho de alto desempenho e desempenho organizacional (GUEST, 1997; ICHNIOWSKI et al., 1996). Na mesma linha, os revisores quantitativos (COMBS et al., 2006; OLIVEIRA, 2006; OLIVEIRA; GONZÁLEZ; OLIVEIRA, 2007; SUBRAMONY, 2009) também concluíram que há uma relação positiva entre GRH e desempenho organizacional. A estimação da magnitude desta relação aumenta quando as práticas de GRH são combinadas em um sistema integrado e coerente, o que de outra maneira já afirmava Deming (1990).

No entanto, com base no alto índice de heterogeneidade apresentado nos resultados, estes revisores apontaram para a possibilidade de que variáveis contextuais estão incidindo sobre a relação GRH-desempenho organizacional. Esta suposição corrobora com a de Jackson, Schuler e Rivero (1989), de que a efetividade de uma prática de GRH é dependente de variáveis contextuais. Por isso, como afirmaram inicialmente Becker e Gerhart (1996) e depois Fields, Chan e Akhtar (2000), para entender a relação entre GRH e desempenho organizacional, essas variáveis, sejam estratégicas, organizacionais e/ou ambientais, não podem ser ignoradas, sob o risco de erros de especificações.

Pelo exposto, a fim de ampliar o entendimento da relação entre GRH e desempenho organizacional e contribuir com argumentos teóricos, reforçados por várias evidências empíricas, que possam promover uma ação mais contextualizada por parte dos Gestores de Operação e Produção, estabelecemos o seguinte objetivo: identificar e desenvolver modelos com variáveis moderadoras de tipo contextual. Para tanto, por meio de uma metanálise, testamos três modelos de variáveis moderadoras de tipo contextual: contexto geográfico, setor de atividade e tipos de empregados. $\mathrm{Na}$ sequência, discorremos sobre cada uma destas variáveis contextuais.

\subsection{Contexto geográfico}

Segundo Jackson e Schuler (1995), a consciência de que há diferenças na forma de gerenciar recursos humanos entre países aumentou com a globalização da economia e o desenvolvimento de empresas multinacionais. Posto que, além do ambiente legal, social e político, cada país possui uma orientação cultural própria. Desse modo, as organizações que atuam ou pretendem atuar globalmente precisam entender as peculiaridades inerentes a cada contexto geográfico, para gerenciar seus recursos humanos de forma efetiva.

Enquanto que para Wright, Snell e Dyer (2005), esta consciência veio com a divulgação dos resultados do estudo de Hofstede, na década de 1980. Esse estudo analisou longitudinalmente os modelos de valores relacionados com trabalho em uma amostra de 116.000 empregados de 50 países diferentes. De acordo com os resultados desse estudo, a variância entre países pode ser explicada por quatro dimensões básicas que são denominadas de: distância de poder; aversão à incerteza; individualismo versus coletivismo; e masculinidade versus feminilidade (HOFSTEDE, $1983 a, b)$.

Estas dimensões têm oferecido uma estrutura teórica para desenvolver hipóteses em estudos de organizações cross-culturais. Por isso, esse estudo é amplamente conhecido para comparar culturas nacionais. Mais especificamente, para promover a premissa de que cada nação é caracterizada por um conjunto único de valores culturais compartilhados, e que é este conjunto que lidera para as diferenças no desenho e, por conseguinte, na eficácia de práticas de GRH entre países.

Tregaskis (1997) analisou comparativamente 531 organizações Francesas e 879 organizações Britânicas e concluiu que o contexto nacional é um forte preditor de como a prática de desenvolvimento de RH é organizada. Ding e Akhtar (2001) sugeriram, com base em um estudo realizado em duas regiões chinesas, que a localização da organização influencia em suas práticas de GRH. Por sua vez, Wang et al. (2003) demonstraram empiricamente que há uma grande diferença cultural, política e econômica, entre a China e os países ocidentais, que interfere em suas relações de emprego.

Paauwe e Boselie (2003) arguiram que as diferenças em envolvimento e em cenário institucional entre países afetam o desenvolvimento da função de GRH. O estudo de caso de McCourt e Ramgutty Wong (2003) no Serviço Civil da Mauritânia e o estudo de Webster e Wood (2005) em Moçambique são bons exemplos disso. Estes estudos demostraram que a efetividade das práticas de GRH é dependente de um contexto institucional e geográfico que suporte sua adoção.

Stavrou-Costea, Charalambous e Spiliotis (2007) também demonstraram empiricamente que o contexto geográfico faz diferença na relação GRH-desempenho. Segundo esses autores, as organizações europeias são menos autônomas do que as organizações norte-americanas, devido principalmente às restrições culturais e legais que as primeiras enfrentam. Diante das evidências empíricas apresentadas, desenvolvemos a hipótese de que:

Hipótese 1: O contexto geográfico da organização analisada modera a relação entre prática de GRH, ou sistema de GRH, e desempenho organizacional.

\subsection{Setor de atividade}

Jackson e Schuler (1995) explicaram que o setor de atividade refere-se às organizações de diferentes grupos de atividades ou setor produtivo. E que, embora muito mais amplo, os setores organizacionais 
podem ser classificados basicamente por uma simples distinção entre organizações agrícolas, organizações de serviços e organizações manufatureiras.

Barrette e Carriere (2003) sugeriram que a natureza diferente das atividades destes tipos de organizações pode repercutir sobre os vários sistemas de GRH. Isto significa que as características que distinguem estes setores podem afetar tanto a escolha quanto o impacto das práticas de GRH sobre o desempenho organizacional. Por este motivo, a desconsideração deste fator contextual em pesquisa de GRH pode limitar suas predições.

Segundo Batt (2002), os processos de trabalho, o nível de interdependência técnica requerida entre os trabalhadores, e o papel dos clientes no processo de produção, dentre inúmeros outros fatores, não podem deixar de ser considerados como fatores contextuais. Por isso, os resultados de estudos realizados em organizações agrícolas, de serviços ou manufatureiras, não podem ser generalizados de um para o outro.

Para efeito deste trabalho, classificamos o setor de atividade em três categorias: setor terciário, setor secundário e setor primário. O setor terciário abrange os serviços em geral. Enquanto que o setor secundário engloba a produção de bens físicos por meio da transformação de matérias-primas, realizada pelo trabalho humano com o auxílio de máquinas e ferramentas. Já o setor primário reúne as atividades agropecuárias e extrativas (vegetais e minerais) (SANDRONI, 1999). No entanto, devido ao fato de que não foram identificados estudos que analisaram organizações no setor primário, só analisamos o setor de atividade para os setores terciário, de serviços; e secundário, de manufatura.

Existem três características básicas que diferem as organizações de serviços das organizações manufatureiras. Na primeira, os produtos das organizações de serviços são intangíveis; a segunda diz respeito ao fato de que os clientes são ativamente envolvidos na produção de serviços; e, na última, o consumo dos serviços ocorre simultaneamente com sua produção (JACKSON; SCHULER, 1995; JACKSON; SCHULER; RIVERO, 1989; REDMAN; MATHEWS, 1998).

Com base nestas características, Cappelli e Crocker Hefter (1996) advogam que a ligação entre práticas de GRH e competitividade organizacional é mais direta em organizações de serviços. Devido ao fato de que os empregados, por eles mesmos, criam o que a organização vende, e o "produto" é o serviço provido diretamente por empregados em interação com os clientes. Em complemento, Ordiz e Fernández (2005) sugeriram, com base em um estudo empírico, que os efeitos positivos das práticas de GRH em organizações de serviços que atuam em ambientes competitivos são maiores do que nas demais organizações.

No entanto, em contrapartida, as metanálises de Combs et al. (2006) e de Subramony (2009) suportaram a hipótese de que os efeitos de sistemas de práticas de GRH são maiores entre organizações manufatureiras do que entre organizações de serviços. Isto se deve ao fato de que as organizações de serviços têm fontes alternativas para desenvolver as competências e a motivação dos empregados, o que reduz os efeitos das práticas de GRH sobre estes outcomes; por sua vez, a participação dos clientes na produção de serviços também limita seus efeitos; ademais, algumas práticas de GRH alinham-se melhor com trabalhos de manufatura; e, por fim, as organizações manufatureiras beneficiam-se mais da flexibilidade destas práticas.

Em suma, as evidências empíricas descritas nos levam a acreditar que o setor de atividade interfere na efetividade das práticas ou sistemas de GRH. Embora haja contradição em relação à afirmação de qual dos dois setores, se o setor de serviços ou o setor de manufatura, é mais favorável para esta efetividade. Desde modo, nos limitamos a predizer que:

Hipótese 2: O setor de atividade da organização analisada modera a relação entre prática de GRH, ou sistema de GRH, e desempenho organizacional.

\subsection{Tipo de empregado}

Schuler e Jackson (1987) investigaram a influência da estratégia de negócios na adoção de práticas de GRH e descobriram adicionalmente, em um estudo com 304 unidades de negócios, que a variação na adoção destas práticas foi maior dentro das mesmas organizações do que entre organizações com relação a sua estratégia de negócios. Por sua vez, Jackson, Schuler e Rivero (1989) analisaram características organizacionais como preditoras de práticas de GRH e encontraram diferenças significativas entre as práticas adotadas para empregados horistas e aquelas adotadas para empregados em nível gerencial.

Isto significa que, provavelmente, as organizações, por meio de seu gerenciamento, utilizam diferentes práticas de GRH para empregados em diferentes níveis. Desta forma, estes resultados demonstraram que os fatores que interferem na adoção de práticas de GRH podem estar relacionados também aos diferentes tipos de empregados que compõem e atuam em uma organização.

Quando Miles e Snow (1984) apresentaram diretrizes para desenvolver sistemas de GRH proativos, também chamaram atenção para o fato de que determinadas práticas de GRH devem ser desenhadas para considerar os requisitos específicos de cada grupo de empregados. Lepak e Snell (1999) reconheceram que cada grupo de empregados possui competências e importância estratégica distintas e desenvolveram quatro configurações de GRH para cada modo de emprego. O argumento de Lepak e Snell $(1999,2002)$ é que não há melhor conjunto de práticas de GRH para todos os empregados dentro de uma organização. Preferencialmente, dentro de uma 
mesma organização, diferentes modos de emprego, bem como diferentes configurações de GRH, podem ser utilizados para gerenciar diferentes grupos de empregados.

Fields, Chan e Akhtar (2000), com base na teoria baseada em recursos e capacidades, sugeriram que as organizações podem investir substancialmente no desenvolvimento e manutenção daqueles empregados vistos como fontes de vantagem competitiva, e investir pouco ou quase nada naqueles empregados vistos como recursos não valiosos, não raros, facilmente imitáveis e substituíveis. Em complemento, Melián-González e Verano-Tacoronte (2006), fundamentados nos conceitos de valor e unicidade estratégica, validaram empiricamente a hipótese de que, dentro de uma mesma organização, as práticas de GRH não são as mesmas para todos os empregados.

Com base nesses argumentos, Lopez-Cabrales, Valle-Cabrera e Herrero (2006) investigaram e confirmaram, em uma amostra de organizações espanholas, que um maior investimento em empregados centrais contribui para a eficiência e a competitividade organizacional. Pelo exposto, o não reconhecimento das diferenças existentes entre grupos de empregados, quanto às suas competências e importância estratégica, pode mascarar aspectos importantes de como organizações gerenciam cada um destes grupos. Portanto, pressupomos que:

Hipótese 3: O tipo de empregado para quem as práticas de GRH foram direcionadas modera a relação entre prática de GRH, ou sistema de GRH, e desempenho organizacional.

\section{Estratégia de busca}

Para compor a nossa amostra, iniciamos uma busca em duas listas de referências de revisões e de bibliografias especializadas (COMBS et al., 2006; OLIVEIRA, 2006). Depois, seguimos a busca em múltiplas bases de dados selecionadas por sua relevância ao tema - Web of Science, Econlit e PsycINFO, Academic Search Premier; a Business Source Premier; Dissertations \& Theses e ABI/ INFORM Global -, nas quais utilizamos os seguintes termos de busca: (human resource management or high performance work practices) and (organizational effectiveness or firm performance), entre os anos de 1980 e 2008. Além disso, selecionamos e adicionamos quatro bases de dados nesta estratégia de busca: Banco de Teses do Portal de Periódicos da CAPES/ MEC e SCIELO Brasil - Scientific Electronic Library Online, para capturar material de língua portuguesa; e ISOC - Ciências Sociales y Humanidades e Tesis Españolas - TESEO, para localizar material de língua espanhola. Nessas bases adicionais utilizamos os termos de busca sugeridos pelas próprias bases. Para complementar e finalizar a estratégia de busca, adotamos o procedimento denominado de Consulta. Ao todo, identificamos 1567 referências diferentes.
Depois, dessa identificação, procedemos a uma análise minuciosa para avaliar a inclusão de cada uma dessas referências neste estudo, de acordo com os seguintes critérios de elegibilidade de: 1) ser o registro de pelo menos um estudo primário de pesquisa empírica; 2) ter em suas medidas uma ou mais de práticas de $\mathrm{GRH}$; 3 ) ter em suas medidas um ou mais indicadores de desempenho organizacional; 4) oferecer em seus resultados informes estatísticos suficientes para o cálculo do tamanho do efeito entre as duas variáveis; 5) ser apresentado em língua inglesa, portuguesa ou espanhola; 6) oferecer dados originais que não se repetem em outro estudo; e 7) ter sido realizado e/ou publicado durante a, ou depois da, década de 1980. Ao final, localizamos o texto completo e selecionamos 191 referências para compor a amostra deste estudo, por atenderem a todos os critérios de elegibilidade. As referências completas das 191 referências selecionadas apresentamos na lista de Bibliografia analisada.

No entanto, identificamos que três registros de estudos, na realidade, apresentavam dados de dois estudos diferentes. O registro de estudo de Allen, Shore e Griffeth (2003) que apresentou separadamente dados de um estudo com 197 empregados de seguros e outro estudo com 215 empregados de vendas de uma loja de departamento. Os registros de estudo de Tzafrir (2005), que foram condensados, apresentaram dados de dois estudos realizados em tempos diferentes e independentes, um em 1996 e o outro em 2000. E o registro de Zacharatos, Barling e Iverson (2005) que apresentou dois estudos com características bem diferentes, quanto à amostra, o tipo de medida de desempenho organizacional utilizado, e o nível de análise. Pelo exposto, dividimos estes três registros em dois cada um. Assim, a partir deste instante, para efeito desta metanálise, ampliamos a amostra de 191 para 194. Dessa forma, essas metanálises foram realizadas com base nos resultados de 194 registros de estudos empíricos e originais, realizados e/ou publicados entre os anos de 1985 a 2008; sobre uma ou mais práticas de GRH e um ou mais indicadores de desempenho organizacional.

\subsection{Codificação}

Para operacionalizar as variáveis, contexto geográfico, setor de atividade e tipo de empregado, conceituamos, dividimos e classificamos cada registro de estudo previamente. O contexto geográfico da organização referiu-se ao local onde o estudo foi realizado. Para operacionalizar esta variável, agrupamos os países de realização do estudo por área geográfica da seguinte forma: América do Norte, Europa, Ásia e Outros. Desta forma, agrupamos os países Estados Unidos e Canadá na categoria América do Norte; Alemanha, Dinamarca, França, Espanha, Reino Unido, Noruega, Itália, Países Baixos, Suécia, 
Grécia, Rússia e Ucrânia na categoria Europa; China, Taiwan, Filipinas, Hong Kong, Japão, Korea, Tailândia, Singapura na categoria Ásia. E, o continente Africano, e os países Austrália, Nova Zelândia, Emirados Árabes, Índia, Israel, Paquistão, Turquia, Vietnam e Jamaica na categoria Outros.

O setor de atividade da organização referiu-se ao conjunto das atividades econômicas, ou setor produtivo da economia. Conforme explicitamos anteriormente, analisamos os registros de estudos que foram realizados nos setores de atividade secundário e terciário, aos quais classificamos e denominamos, respectivamente, de organizações manufatureiras e de organizações de serviços.

Por fim, a variável contextual tipo de empregado foi operacionalizada quanto ao direcionamento das práticas de GRH analisado em cada estudo, se: para um grupo específico ou mais de um grupo de empregados; ou para todos os empregados indiscriminadamente. Desta forma, classificamos cada registro de estudo em duas categorias discriminado ou indiscriminado.

Avaliamos a confiabilidade intercodificadores dado por dado, de forma independente. Ademais, seguindo as diretrizes de Orwin (1994), para cada dado, utilizamos três índices específicos de confiabilidade intercodificadores (IRR): o índice de acordo (AR), o Kappa de Cohen (k) e a correlação intercodificadores (r). Para os índices k e r; e arbitramos um valor mínimo para AR. Deste modo, consideramos AR excelente quando seu valor foi igual ou superior a ,90; consideramos $\mathrm{k}$ excelente quando foi estimado igual ou acima de ,75; e consideramos $r$ adequado quando seu valor foi igual ou superior a ,800. Na sequência, o resultado desta avaliação para os dados codificados.

Para os dados sobre o contexto geográfico, não foi necessário calcular nenhum IRR, tendo em vista que houve um consenso total. Já os dados sobre o setor de atividade, os índices de confiabilidade intercodificadores foram: $\mathrm{AR}=, 92 ; \mathrm{k}=, 88 \mathrm{e} \mathrm{r}=, 940$. Consideramos todos estes índices excelentes. Em relação aos dados sobre o tipo de empregado, os índices foram: $\mathrm{AR}=, 87 ; \mathrm{k}=, 69 \mathrm{e} \mathrm{r}=, 726$. Embora nenhum dos índices tenha apresentado um resultado excelente, consideramos estes índices dentro de um nível satisfatório. Em linhas gerais, os índices de confiabilidade entre codificadores sugerem que a estratégia de codificação foi satisfatória. Depois desta análise, para cada dado em desacordo, independente de qual tenha sido o índice de confiabilidade intercodificadores estimado, os dois codificadores retornaram aos textos completos dos estudos e decidiram os desacordos por consenso.

\subsection{Procedimentos Meta-Analíticos}

De acordo com Hunter e Schmidt (2004), uma variável moderadora pode ser revelada de duas formas dependentes: 1) quando a média da correlação varia de subagrupamento para subagrupamento; e 2) quando a variância corrigida mediará menor nos subagrupamentos que para os dados como um todo. Isto significa que, se a média da correlação varia por subagrupamentos [(Ave(p))], então a média do desvio padrão (AveSDp) dos subagrupamentos deve ser menor do que o desvio padrão no conjunto de dados combinado (SDp).

Ademais, para testar variáveis moderadoras, Hunter e Schmidt (2004) recomendaram que fosse feita a comparação do mean da correlação de cada subagrupamento, pelo intervalo de confiança. Assim, para estimar o intervalo de confiança do mean da correlação da população real, (p), tivemos que calcular o erro padrão (SE). Para tanto, utilizamos a fórmula sugerida por Hunter e Schmidt (2004): $\mathrm{SE}=\operatorname{raiz}\left(\sigma \mathrm{r}^{2} / \mathrm{k}\right)$.

Nestes casos, depois da estimação do intervalo de confiança, verificamos, por meio de representação gráfica, se entre os means de cada subagrupamento havia sobreposição de valores tanto no limite mínimo quanto no limite máximo. Em caso afirmativo, descartamos a variável previamente hipotetizada como moderadora. Em caso negativo, consideramos a variável previamente hipotetizada como moderadora.

Por fim, para complementarmos a análise das variáveis moderadoras, para as variáveis setor de atividade e tipo de empregado, nas quais foi possível a comparação entre dois grupos, calculamos o mean padronizado da diferença do tamanho do efeito (g). Para interpretar este valor, seguimos a regra de Cohen. Desse modo, interpretamos a diferença entre os grupos como pequena quando o valor calculado de $g$ foi igual ou menor do que ,200. Consideramos a diferença entre os grupos entre pequena e média quando o valor de $\mathrm{g}$ foi maior do que ,200 e menor do que ,500. Enquanto que interpretamos como uma diferença entre os grupos entre média e grande quando o valor de g foi maior do que ,500 e menor do que ,800. E, por fim, consideramos uma grande diferença entre os grupos quando o valor de $\mathrm{g}$ foi maior do que ,800 (HOWELL, 2010).

\section{Resultados}

Para realizarmos esta análise, utilizamos as médias dos coeficientes de correlação de Pearson extraídos em cada registro de estudo. Trinta e quatro estudos mediram e apresentaram dados tanto de práticas independentes quanto de sistemas de GRH. Para evitar dependência de dados, separamos estes estudos comuns em dois agrupamentos diferentes. Assim, destinamos um agrupamento, ao qual denominamos de agrupamento A, para todos os estudos que mediram sistemas de GRH. Este agrupamento rendeu 108 correlações, sendo 74 correlações de estudos que só mediram sistemas de GRH e 34 correlações entre sistemas de GRH e desempenho organizacional 
extraídas dos estudos comuns composto por todos os estudos que mediram sistemas de GRH. Especificamente, para a variável tipo de empregado, além das 108 correlações, acrescentamos mais uma correlação, devido ao estudo de Fey, Bjorkman e Pavlovskaya (2000) que apresentou dados separados para empregados em nível gerencial e empregados em nível não gerencial.

Enquanto que destinamos outro agrupamento, ao qual denominamos de agrupamento $\mathrm{B}$, para todos os estudos que mediram práticas de GRH separadas. Este agrupamento constituiu-se de 120 correlações, 86 correlações de estudos que só avaliaram práticas de GRH separadas e 34 de correlações extraídas de práticas de GRH e desempenho organizacional nos estudos comuns. Também para a variável tipo de empregados, adicionamos seis correlações, devido aos estudos que mediram e apresentaram dados para dois tipos de empregados diferentes (FEY; BJORKMAN; PAVLOVSKAYA, 2000; LUTHANS; PETERSON, 2003; MAK; AKHTAR, 2003; SANZ-VALLE; SÁNCHEZ, 2002; STAHL, 2000; STOREY, 2002).

Por fim, para possibilitar uma análise geral, preparamos mais um agrupamento, denominado de agrupamento $\mathrm{C}$, unindo as correlações dos outros dois agrupamentos. No entanto, para garantir a interdependência de dados, excluímos as correlações dos 34 estudos que apresentaram resultados tanto de práticas isoladas de GRH quanto de sistemas de GRH. Dessa maneira, este agrupamento finalizou com 160 estudos.

Tanto o agrupamento A quanto o agrupamento $\mathrm{B}$ atenderam aos requisitos de heterogeneidade necessários para justificar a metanálise de grupos de categorias de variáveis e testar o efeito moderador de algumas variáveis previamente hipotetizadas. Para o agrupamento $\mathrm{A}$, o $\mathrm{S}^{2}$ equivale a $87 \%$ da $\sigma^{2} \mathrm{r}$; o $\mathrm{SDp}$; ,165 é maior do que $1 / 4$ do mean da população; ,059; e o valor de Q, 1.080 é significativo. Enquanto que para o agrupamento $\mathrm{B}, \mathrm{o} \mathrm{S} 1^{2}$ equivale a $95 \%$ da $\sigma^{2} \mathrm{r}$; o SDp; , 161 é maior do que $1 / 4$ do mean da população; ,037; e ,para finalizar, o valor de Q, 2.040 também é significativo. Adicionalmente, os dados gerais do agrupamento $\mathrm{C}$ também atendem aos requisitos de heterogeneidade, necessários para justificar a busca por efeitos moderadores. Sua variância residual $\left(\mathrm{S}^{2}\right)$ equivale a $93 \%$ da variância total $\left(\sigma^{2} \mathrm{r}\right)$; o SDp;, 168 é maior do que $1 / 4$ do mean da população; ,041; e o valor de Q, 3.200 é significativo.

Estes dados permitiram a continuação de nossa análise. Assim, para atendermos ao objetivo de identificar e desenvolver modelos com variáveis moderadoras de tipo contextual, realizamos procedimentos específicos referentes a cada uma das variáveis contextuais hipotetizadas como variáveis moderadoras. No restante desta secção, apresentaremos os resultados das metanálises realizadas para cada variável e cada agrupamento, seja A, B ou C.

Para a variável contexto geográfico, no agrupamento A, o maior mean foi para Outros $(\mathrm{k}=10 ; \mathrm{p}=, 482$; $\alpha \leq, 200)$, seguido por Ásia $(\mathrm{k}=23 ; \mathrm{p}=, 364$; $\alpha \leq, 100)$, Europa $(\mathrm{k}=31 ; \mathrm{p}=, 201$; ns), e, por último, América do Norte ( $\mathrm{k}=40 ; \mathrm{p}=, 175 ; \mathrm{ns})$. O Ave(SDp) dos quatro grupos foi menor; , 146, do que o SDp; ,165. No entanto, houve uma sobreposição de dados significativa entre os means dos grupos Europa e América do Norte.

Os resultados para o agrupamento B foram similares. O maior mean também foi para Outros ( $\mathrm{k}=13 ; \mathrm{p}=, 367 ; \mathrm{ns})$, seguido por Ásia $(\mathrm{k}=14$; $\mathrm{p}=, 292 ; \mathrm{ns})$, Europa $(\mathrm{k}=29 ; \mathrm{p}=, 210 ; \mathrm{ns}), \mathrm{e}$, novamente por último, América do Norte $(\mathrm{k}=57$; $\mathrm{p}=, 109 ; \mathrm{ns})$. O Ave(SDp) dos quatro grupos foi menor; ,158, do que o SDp; ,199. No entanto, não houve sobreposição de dados entre os means de nenhum dos grupos. Adicionalmente, a diferença de means entre os grupos Ásia e América do Norte - que são comparativamente os dois grupos mais diferentes, tanto para o agrupamento A quanto para o agrupamento $\mathrm{B}$ - foi considerada grande (respectivamente, $\mathrm{g}=1,145$ e $g=, 920$ ). Deste modo, embora a sobreposição de dados significativa entre os means dos grupos Europa e América do Norte, no agrupamento A, os dados das metanálises dos agrupamentos A e B indicam a possibilidade de um efeito moderador da variável contexto geográfico.

Confirmamos essa possibilidade com os resultados da metanálise do agrupamento C. Sendo que, novamente, o maior mean foi para o grupo Outros $(\mathrm{k}=19 ; \mathrm{p}=, 453 ; \alpha \leq, 100)$, seguido por Ásia $(\mathrm{k}=29$; $\mathrm{p}=, 326 ; \alpha \leq, 100)$, Europa $(\mathrm{k}=34 ; \mathrm{p}=, 236 ; \alpha \leq, 200)$ e América do Norte $(\mathrm{k}=69 ; \mathrm{p}=, 110 ; \mathrm{ns})$. O Ave(SDp) dos quatro grupos foi menor; ,089, do que o SDp; ,199. E, no gráfico apresentado na Figura 1, podemos

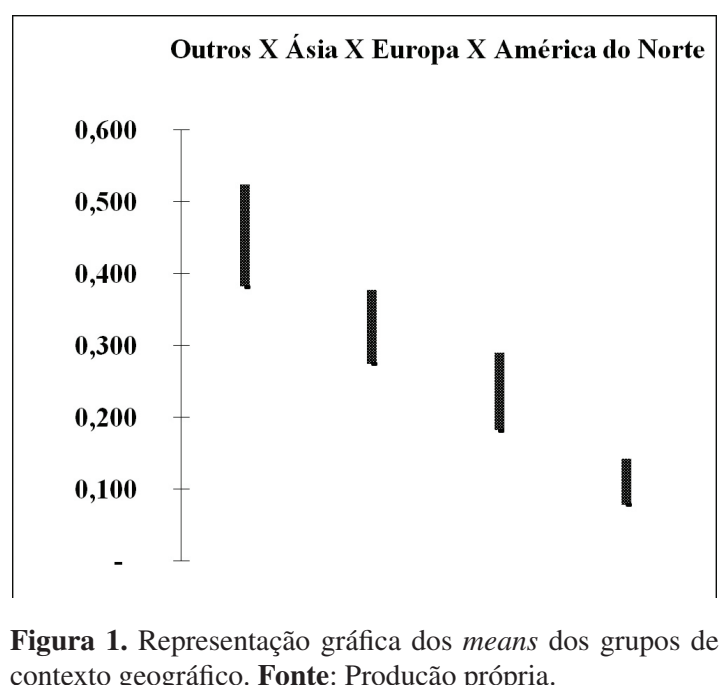
contexto geográfico. Fonte: Produção própria. 


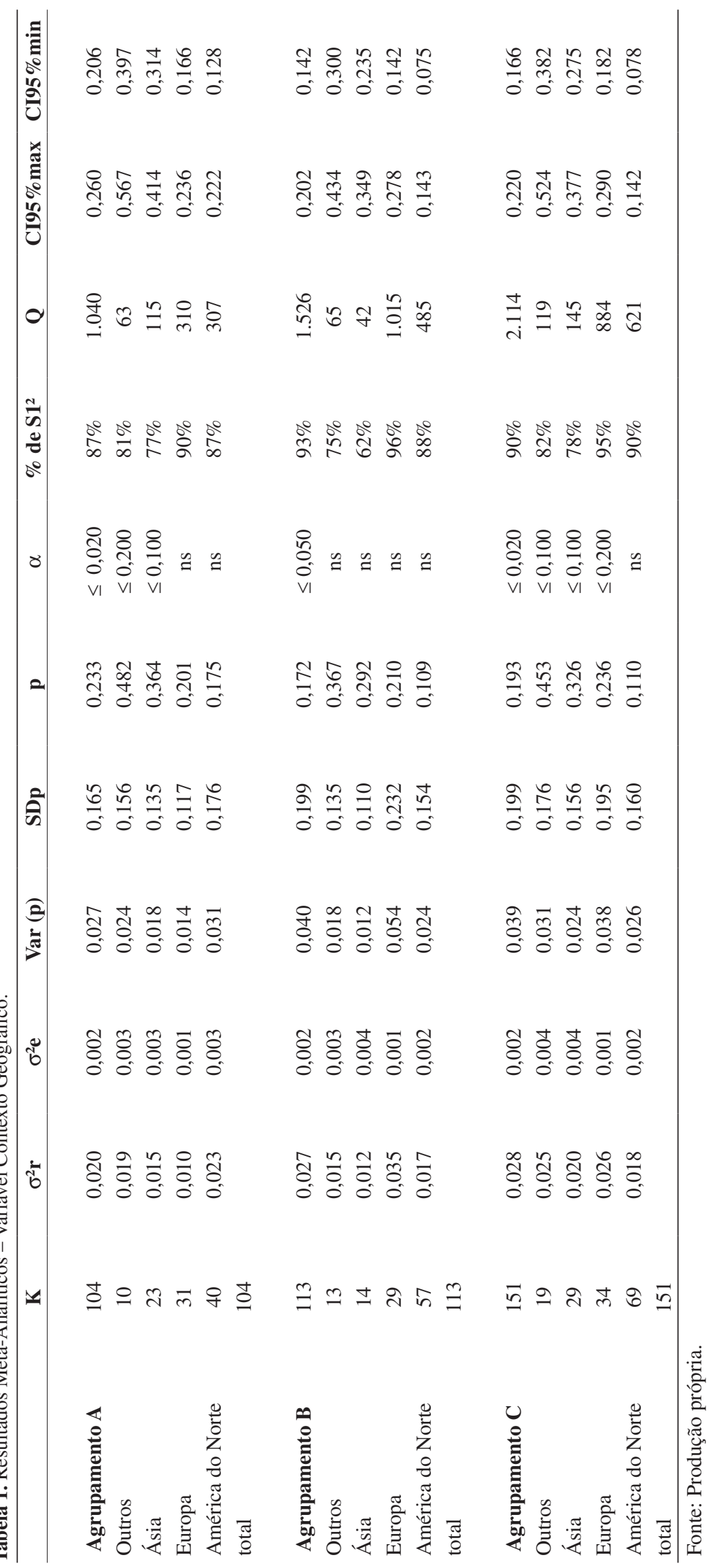


verificar que não houve sobreposição de dados entre os means dos grupos.

Em resumo, os dados das metanálises dos três agrupamentos corroboram com a primeira hipótese. Assim, confirmamos a hipótese de que o contexto geográfico da organização analisada modera a relação entre prática de GRH, ou sistema de GRH, e desempenho organizacional. Na Tabela 1, oferecemos um resumo dos principais dados estatísticos extraídos das três metanálises, para todos os agrupamentos.

No tocante à variável setor de atividade, para ambos os agrupamentos, A e B, os resultados revelaram que o mean de organizações manufatureiras superou

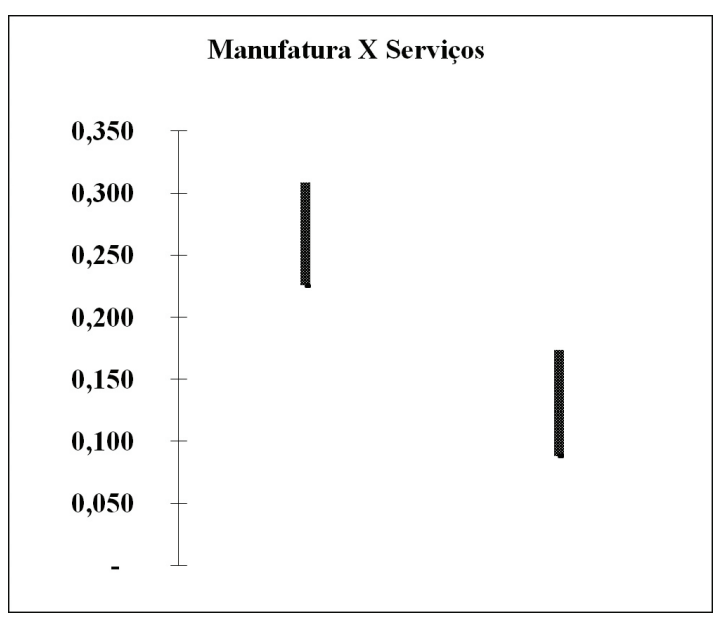

Figura 2. Representação gráfica dos means dos grupos setor de atividade. Fonte: Produção própria. significativamente o mean de organizações de serviços. No agrupamento A, os resultados foram, respectivamente, $\mathrm{k}=29 ; \mathrm{p}=, 336 ; \alpha \leq, 100$ e $\mathrm{k}=19$; $\mathrm{p}=, 263 ; \mathrm{ns}$, com uma diferença de means considerada mediana $(\mathrm{g}=, 406)$. Enquanto que no agrupamento $\mathrm{B}$, os resultados foram, respectivamente, $\mathrm{k}=34$; $\mathrm{p}=, 256 ; \alpha \leq, 200$ e $\mathrm{k}=33 ; \mathrm{p}=, 121 ; \mathrm{ns}$, com uma diferença de means considerada entre mediana e grande $(\mathrm{g}=, 771)$.

Ademais, os Ave(SDp) dos dois grupos, nos dois agrupamentos, foi menor; respectivamente; ,119 e ,162, do que o SDp, respectivamente; , 180 e ,175. A comparação dos means, para o agrupamento $\mathrm{A}$, revelou uma sobreposição apenas entre o limite mínimo de organizações manufatureiras com o limite máximo de organizações de serviços. E, para o agrupamento $\mathrm{B}$, não revelou nenhuma sobreposição de dados.

Por sua vez, os resultados do agrupamento $\mathrm{C}$ confirmaram que o mean de organizações manufatureiras $(\mathrm{k}=47 ; \mathrm{p}=, 267 ; \alpha \leq, 100)$ foi maior do que o mean de organizações de serviços $(\mathrm{k}=40 ; \mathrm{p}=, 131 ; \mathrm{ns})$, com uma diferença de means bem significativa $(g=, 773)$. Ademais, o Ave(SDp) dos dois grupos foi menor; ,165, do que o SDp; ,176. E, como exposto na Figura 2, os means dos dois grupos não apresentaram sobreposição de dados.

Em suma, todos os dados apresentados nas três metanálises nos conduzem para a afirmação de que o setor de atividade da organização analisada modera a relação entre prática de GRH, ou sistema de GRH, e desempenho organizacional. Assim, confirmamos a segunda hipótese que esboçava justamente esta afirmação. Na Tabela 2, apresentamos os principais dados estatísticos extraídos das metanálises dos três agrupamentos.

Tabela 2. Resultados Meta-Analíticos - Variável Setor de Atividade.

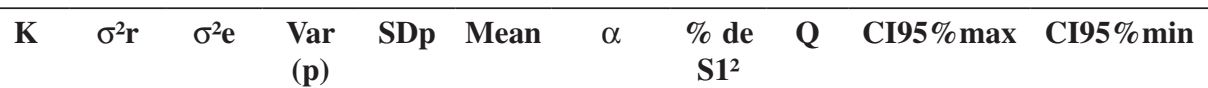

\begin{tabular}{|c|c|c|c|c|c|c|c|c|c|c|c|}
\hline Agrupamento A & 48 & 0,025 & 0,003 & 0,033 & 0,180 & 0,308 & $\leq 0,050$ & $85 \%$ & 400 & 0,353 & 0,263 \\
\hline manufatura & 29 & 0,023 & 0,003 & 0,030 & 0,172 & 0,336 & $\leq 0,100$ & $84 \%$ & 222 & 0,391 & 0,281 \\
\hline serviços & 19 & 0,026 & 0,004 & 0,034 & 0,185 & 0,263 & ns & $84 \%$ & 124 & 0,336 & 0,190 \\
\hline total & 48 & & & & & & & & & & \\
\hline Agrupamento B & 67 & 0,021 & 0,002 & 0,031 & 0,175 & 0,169 & $\leq 0,100$ & $92 \%$ & 704 & 0,204 & 0,134 \\
\hline manufatura & 34 & 0,018 & 0,002 & 0,025 & 0,158 & 0,256 & $\leq 0,200$ & $86 \%$ & 306 & 0,301 & 0,211 \\
\hline serviços & 33 & 0,018 & 0,001 & 0,027 & 0,165 & 0,121 & ns & $93 \%$ & 594 & 0,167 & 0,075 \\
\hline total & 67 & & & & & & & & & & \\
\hline Agrupamento C & 87 & 0,022 & 0,002 & 0,031 & 0,176 & 0,176 & $\leq 0,200$ & $90 \%$ & 957 & 0,207 & 0,145 \\
\hline manufatura & 47 & 0,021 & 0,003 & 0,027 & 0,165 & 0,267 & $\leq 0,100$ & $84 \%$ & 329 & 0,308 & 0,226 \\
\hline serviços & 40 & 0,019 & 0,001 & 0,027 & 0,164 & 0,131 & ns & $92 \%$ & 760 & 0,174 & 0,088 \\
\hline total & 87 & & & & & & & & & & \\
\hline
\end{tabular}

Fonte: Produção própria. 
Por último, em relação à variável tipo de empregado, os resultados para o agrupamento A revelaram que o mean para o grupo discriminado $(\mathrm{k}=33 ; \mathrm{p}=, 242$; $\alpha \leq, 200)$ foi sutilmente maior do que o mean para o grupo indiscriminado $(\mathrm{k}=76 ; \mathrm{p}=, 229 ; \alpha \leq, 050)$. Isto significa que a diferença padronizada dos means dos dois grupos foi insignificante $(g=, 079)$. Embora o Ave(SDp) dos dois grupos; , 160, tenha sido menor do que o SDp; ,165; o intervalo de confiança dos means dos dois grupos apresentou uma sobreposição de dados quase que total.

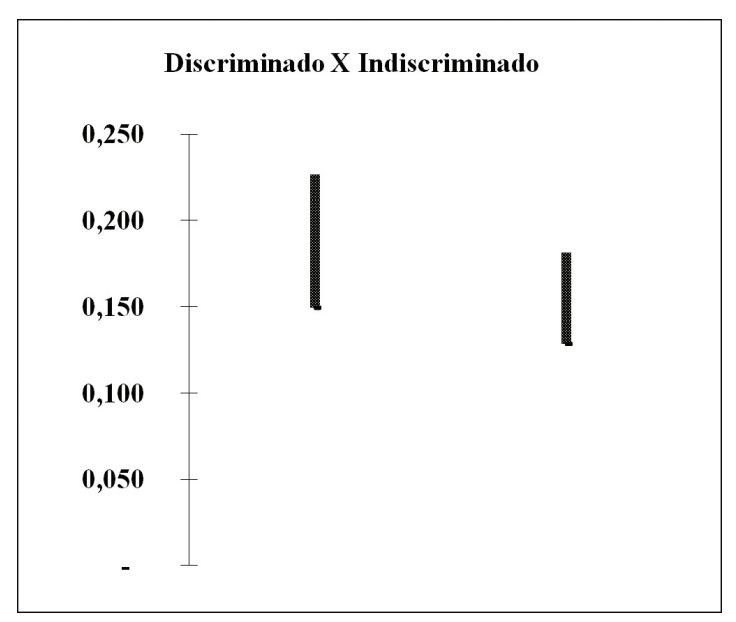

Figura 3. Representação gráfica dos means dos grupos tipo de empregados. Fonte: Produção própria.
Estes dados foram reforçados com os resultados do agrupamento B. Para o agrupamento B, o mean para o grupo discriminado $(\mathrm{k}=48 ; \mathrm{p}=, 161 ; \mathrm{ns})$ também foi maior do que o mean para o grupo indiscriminado $(\mathrm{k}=76 ; \mathrm{p}=, 146 ; \alpha \leq, 200)$. No entanto, a diferença padronizada dos means dos dois grupos foi insignificante $(\mathrm{g}=, 092)$. Ademais, o Ave(SDp) dos dois grupos; , 173, foi maior do que o SDp; ,163; e o intervalo de confiança dos means dos dois grupos apresentou uma total sobreposição de dados.

Adicionalmente, os resultados do agrupamento C, mostraram que a diferença padronizada do mean do grupo discriminado $(\mathrm{k}=59 ; \mathrm{p}=, 188 ; \alpha \leq, 200) \mathrm{em}$ relação ao mean do grupo indiscriminado $(\mathrm{k}=104$; $\mathrm{p}=, 155 ; \alpha \leq, 100)$ foi pequena $(\mathrm{g}=, 195)$. O Ave(SDp) dos dois grupos; , 172, foi maior do que o SDp; ,169. E a representação gráfica do intervalo de confiança dos dois grupos, como podemos comprovar com a Figura 3, apresentou uma grande sobreposição de dados.

Os dados das metanálises realizadas com os agrupamentos A e B já foram conclusivos para a não confirmação da terceira hipótese. $\mathrm{E}$ os dados da metanálise realizada com o agrupamento C só reforçaram que os dados apresentados não oferecem indicativos de variável moderadora. Destarte, a terceira hipótese, na qual predissemos que o tipo de empregado para quem as práticas de GRH foram direcionadas modera a relação entre prática de GRH, ou sistema de GRH, e desempenho organizacional, não foi confirmada. Na Tabela 3, apresentamos os principais resultados das metanálises dos agrupamentos A, B e C.

Tabela 3. Resultados Meta-Analíticos - Variável Tipo de Empregado.

\begin{tabular}{|c|c|c|c|c|c|c|c|c|c|c|c|}
\hline & $\mathbf{K}$ & $\sigma^{2} \mathbf{r}$ & $\sigma^{2} e$ & $\begin{array}{l}\text { Var } \\
\text { (p) }\end{array}$ & SDp & Mean & $\alpha$ & $\begin{array}{c}\% \text { de } \\
\mathrm{S1}^{2}\end{array}$ & $\mathbf{Q}$ & CI95\% max & CI95\% $\mathrm{min}$ \\
\hline Agrupamento A & 109 & 0,020 & 0,002 & 0,027 & 0,165 & 0,234 & $\leq 0,020$ & $87 \%$ & 1.090 & 0,261 & 0,207 \\
\hline discriminado & 33 & 0,014 & 0,002 & 0,019 & 0,138 & 0,242 & $\leq 0,200$ & $87 \%$ & 231 & 0,282 & 0,202 \\
\hline indiscriminado & 76 & 0,024 & 0,003 & 0,033 & 0,181 & 0,229 & $\leq 0,050$ & $89 \%$ & 608 & 0,264 & 0,194 \\
\hline total & 109 & & & & & & & & & & \\
\hline Agrupamento B & 124 & 0,018 & 0,001 & 0,026 & 0,163 & 0,150 & $\leq 0,100$ & $90 \%$ & 2.232 & 0,174 & 0,126 \\
\hline discriminado & 48 & 0,025 & 0,002 & 0,037 & 0,192 & 0,161 & ns & $92 \%$ & 600 & 0,206 & 0,116 \\
\hline indiscriminado & 76 & 0,016 & 0,001 & 0,023 & 0,153 & 0,146 & $\leq 0,200$ & $89 \%$ & 1.216 & 0,174 & 0,118 \\
\hline total & 124 & & & & & & & & & & \\
\hline Agrupamento C & 163 & 0,020 & 0,001 & 0,029 & 0,169 & 0,165 & $\leq 0,050$ & $92 \%$ & 3.260 & 0,187 & 0,143 \\
\hline discriminado & 59 & 0,023 & 0,002 & 0,032 & 0,179 & 0,188 & $\leq 0,200$ & $92 \%$ & 679 & 0,227 & 0,149 \\
\hline indiscriminado & 104 & 0,019 & 0,001 & 0,027 & 0,164 & 0,155 & $\leq 0,100$ & $93 \%$ & 1.976 & 0,181 & 0,129 \\
\hline total & 163 & & & & & & & & & & \\
\hline
\end{tabular}

Fonte: Produção própria. 


\section{Conclusões}

Identificamos e desenvolvemos modelos com variáveis moderadoras de tipo contextual com base em três diferentes agrupamentos, A, B e C. Ou seja, verificamos cada variável hipotetizada por meio de três metanálises. Das três variáveis previamente hipotetizadas de tipo contextual verificadas, confirmamos que as variáveis setor de atividade e contexto geográfico são variáveis moderadoras. Por outro lado, não confirmamos a hipótese de que a variável tipo de empregado seja uma variável moderadora da relação GRH-desempenho organizacional.

A identificação da variável setor de atividade como uma variável moderadora corrobora com os resultados apresentados nas metanálises de Combs et al. (2006) e de Subramony (2009). Evidenciou-se, também, que a magnitude da relação entre GRH e desempenho é maior entre as organizações manufatureiras do que entre as organizações de serviços.

A comprovação da variável contexto geográfico como uma variável moderadora parece ser algo inédito, tendo em vista que não havia sido testada por nenhuma das metanálises realizadas e publicadas previamente. Contudo, confirmamos a premissa de Hofstede (1983a, b) de que as diferenças no desenho e, por conseguinte, na eficácia das práticas de GRH depende do conjunto único de valores culturais que caracterizam cada país.

Em contrapartida, a não confirmação da variável tipo de empregado como variável moderadora sugere que a magnitude da relação entre práticas de GRH e desempenho organizacional independe de para quem estas práticas estão sendo direcionadas. Com base neste resultado, nos confrontamos com as evidências apresentadas por Lopez-Cabrales, Valle-Cabrera e Herrero (2006) de que um maior investimento em empregados centrais contribui para a eficiência e a competitividade organizacional. Além disso, levanta um questionamento quanto aos critérios para classificar empregados valiosos, raros, difíceis de serem imitáveis e insubstituíveis.

Em suma, com base nos resultados, identificamos que a magnitude da relação entre GRH e desempenho organizacional é moderada pelas variáveis contextuais: setor de atividade e contexto geográfico. No entanto, independe do tipo de empregado para o qual as práticas de GRH estejam sendo direcionadas. Com estes resultados, esperamos ter contribuído com o entendimento desta relação. Mais especificamente, no âmbito desta publicação, esperamos também ter sensibilizado os Gestores de Operação e Produção a considerarem os fatores contextuais em suas práticas organizacionais.

No entanto, como podemos observar nos dados apresentados para cada variável, os resultados ainda apresentaram um alto índice de heterogeneidade.
Isso significa que, além das já identificadas, outras variáveis moderadoras também podem está incidindo sobre a relação GRH-desempenho organizacional. Com isto em mente, apontamos para a necessidade de mais estudos metanalíticos que testem não somente outros modelos de variáveis moderadoras de tipo contextual, mas também de tipo metodológico.

\section{Referências}

ALLEN, D. G.; SHORE, L. M.; GRIFFETH, R. W. The role of perceived organizational support and supportive human resource practices in the turnover process. Journal of Management, v. 29, n. 1, p. 99-118, 2003. http:// dx.doi.org/10.1177/014920630302900107

ARTHUR, J. B. Effects of human resource systems on manufacturing performance and turnover. Academy of Management Journal, v. 37, n. 3, p. 670-687, 1994. http://dx.doi.org/10.2307/256705

BARRETTE, J.; CARRIERE, J. La performance organisationnelle et la complémentarité des pratiques de gestion des ressources humaines. Relations Industrielles, v. 58, n. 3, p. 427-453, 2003. http:// dx.doi.org/10.7202/007494ar

BATT, R. Managing customer services: human resource practices, quit rates, and sales growth. Academy of Management Journal, v. 45, n. 3, p. 587-597, 2002. http://dx.doi.org/10.2307/3069383

BECKER, B. E.; GERHART, B. The impact of human resource management on organizational performance: progress and prospects. Academy of Management Journal, v. 39, n. 4, p. 779-801, 1996. http://dx.doi. org/10.2307/256712

CAPPELLI, P.; CROCKER HEFTER, A. Distinctive human resources are firms' core competencies. Organizational Dynamics, v. 24, n. 3, p. 7-22, 1996.

COMBS, J. et al. How much do high-performance work practices matter? A meta-analysis of their effects on organizational performance. Personnel Psychology, v. 59, n. 3, p. 501-528, 2006. http://dx.doi. org/10.1111/j.1744-6570.2006.00045.x

DELANEY, J. T.; HUSELID, M. A. The impact of human resource management practices on perceptions of organizational performance. Academy of Management Journal, v. 39, n. 4, p. 949-969, 1996. http://dx.doi. org/10.2307/256718

DELERY, J. E.; DOTY, D. H. Modes of theorizing in strategic human resource management: tests of universalistic, contingency, and configurational performance predictions. Academy of Management Journal, v. 39, n. 4, p. 802-835, 1996. http://dx.doi.org/10.2307/256713

DEMING, W. E. Qualidade: a revolução da administração. São Paulo: Marques Saraiva, 1990. PMCid:PMC184425

DING, D. Z.; AKHTAR, S. The organizational choice of human resource management practices: a study of Chinese enterprises in three cities in the PRC. International Journal of Human Resource Management, v. 12, n. 6 , p. 946-964, 2001. http://dx.doi.org/10.1080/713769691

FEY, C. F.; BJORKMAN, I.; PAVLOVSKAYA, A. The effect of human resource management practices on firm performance in Russia. International Journal of 
Human Resource Management, v. 11, n. 1, p. 1-18, 2000. http://dx.doi.org/10.1080/095851900339963

FIELDS, D.; CHAN, A.; AKHTAR, S. Organizational context and human resource management strategy: a structural equation analysis of Hong Kong firms. International Journal of Human Resource Management, v. 11, n. 2, p. 264-277, 2000. http:// dx.doi.org/10.1080/095851900339864

GUEST, D. E. Human resource management and performance: a review and research agenda. International Journal of Human Resource Management, v. 8, n. 3, p. 263-276. 1997.

HOFSTEDE, G. The cultural relativity of organizational practices and theories. Journal of International Business Studies, v. 14, n. 2, p. 75-89, 1983a. http://dx.doi. org/10.1057/palgrave.jibs.8490867

HOFSTEDE, G. National cultures in four dimensions: a research-based theory of cultural difference among nations. International Studies of Management \& Organization, v. 13, n. 1-2, p. 46-74, 1983 b.

HOWELL, D. C. Statistical methods for psychology. 7. ed. Australia: Cengage Learning, 2010.

HUNTER, J. E.; SCHMIDT, F. L. Methods of metaanalysis: correcting error and bias in research findings. 2. ed. Thousand Oaks: Sage Publications, 2004.

HUSELID, M. A. The impact of human resource management practices on turnover, productivity, and corporate financial performance. Academy of Management Journal, v. 38, n. 3, p. 635-672, 1995. http://dx.doi.org/10.2307/256741

HUSELID, M. A.; JACKSON, S. E.; SCHULER, R. S. Technical and strategic human resource management effectiveness as determinants of firm performance. Academy of Management Journal, v. 40, n. 1, p. 171-188, 1997. http://dx.doi.org/10.2307/257025

ICHNIOWSKI, C. et al. What works at work: overview and assessment. Industrial Relations, v. 35, n. 3, p. 299-333, 1996. http://dx.doi.org/10.1111/j.1468-232X.1996. tb00409.x

JACKSON, S. E.; SCHULER, R. S. Understanding human resource management in the context of organizations and their environments. Annual Review of Psychology, v. 46, p. 237-264, 1995. PMid:19245335. http://dx.doi. org/10.1146/annurev.ps.46.020195.001321

JACKSON, S. E.; SCHULER, R. S.; RIVERO, J. C. Organizational characteristics as predictors of personnel practices. Personnel Psychology, v. 42, n. 4, p. 727-786, 1989. http://dx.doi.org/10.1111/j.1744-6570.1989. tb00674.x

JABBOUR, C. J. C. et al. Gestão de recursos humanos e desempenho operacional: evidências empíricas. Gestão \& Produção, v. 19, n. 2, p. 347-360, 2012. http://dx.doi. org/10.1590/S0104-530X2012000200009

LACOMBE, B. M. B. Avaliação de resultados em RH: análise dos métodos propostos na literatura acadêmica. São Paulo: FGV-EAESP, 2004. (Relatório de Pesquisa, n. 29).

LEPAK, D. P.; SNELL, S. A. The human resource architecture: toward a theory of human capital allocation and development. Academy of Management Review, v. 24, n. 1, p. 31-48, 1999.
LEPAK, D. P.; SNELL, S. A. Examining the human resource architecture: the relationships among human capital, employment, and human resource configurations. Journal of Management, v. 28, n. 4, p. 517-543, 2002. http:// dx.doi.org/10.1016/S0149-2063(02)00142-3

LOPEZ-CABRALES, A.; VALLE-CABRERA, R.; HERRERO, I. The contribution of core employees to organizational capabilities and efficiency. Human Resource Management, v. 45, n. 1, p. 81-109, 2006. http://dx.doi.org/10.1002/hrm.20094

LUTHANS, F.; PETERSON, S. J. 360-degree feedback with systematic coaching: Empirical analysis suggests a winning combination. Human Resource Management, v. 42, n. 3, p. 243-256, 2003. http://dx.doi.org/10.1002/ hrm. 10083

MACDUFFIE, J. P. Human resource bundles and manufacturing performance: organizational logic and flexible production systems in the world auto industry. Industrial and Labor Relations Review, v. 48, n. 2, p. 197-221, 1995. http://dx.doi.org/10.2307/2524483

MAK, S. K. M.; AKHTAR, S. Human resource management practices, strategic orientations, and company performance: a correlation study of publicly listed companies. Journal of American Academy of Business, v. 2, n. 2, p. 510-515, 2003.

MCCOURT, W.; RAMGUTTY WONG, A. Limits to strategic HRM: the case of the Mauritian civil service. International Journal of Human Resource Management, v. 14, n. 4, p. 600-618, 2003. http:// dx.doi.org/10.1080/0958519032000057790

MELIÁN-GONZÁLEZ, S.; VERANO-TACORONTE, D. Is there more than one way to manage human resources in companies? Personnel Review, v. 35, n. 1, p. 29-50, 2006. http://dx.doi.org/10.1108/00483480610636777

MILES, R. E.; SNOW, C. C. Designing strategic human resources systems. Organizational Dynamics, v. 13, n. 1, p. 36-52, 1984. http://dx.doi. org/10.1016/0090-2616(84)90030-5

OLIVEIRA, A. M. B. D. A relação entre as práticas de recursos humanos e o desempenho organizacional: uma metanálise. 2006. 185 f. Trabalho de Conclusão de Curso-Departamento de Psicología Social y Antropología, Universidad de Salamanca, Salamanca, 2006.

OLIVEIRA, A. M. B. D.; GONZÁLEZ, L. F.; OLIVEIRA, A. J. D. The design investigation as a variable moderator in the study of the relationship between practices of HR and organizational performance. In: EUROPEAN CONGRESS OF WORK AND ORGANIZATIONAL PSYCHOLOGY, 13., 2007, Stockholm. Anais...

ORDIZ, M. F.; FERNÁNDEZ, E. Influence of the sector and the environment on human resource practices' effectiveness. International Journal of Human Resource Management, v. 16, n. 8, p. 1349-1373, 2005. http://dx.doi.org/10.1080/09585190500220242

ORWIN, R. G. Evaluating coding decisions. In: COOPER, H.; HEDGES, L. V. (Eds.). The handbook of research synthesis. New York: Russell Sage Foundation, 1994. p. 139-162.

PAAUWE, J.; BOSELIE, P. Challenging 'strategic HRM' and the relevance of the institutional setting. Human Resource Management Journal, v. 13, n. 3, p. 56-70, 
2003. http://dx.doi.org/10.1111/j.1748-8583.2003. tb00098.x

REDMAN, T.; MATHEWS, B. P. Service quality and human resource management: a review and research agenda. Personnel Review, v. 27, n. 1, p. 57-77, 1998. http:// dx.doi.org/10.1108/00483489810368558

ROGERS, E. W.; WRIGHT, P. M. Measuring organizational performance in strategic human resource management: problems, prospects, and performance information markets. Human Resource Management Review, v. 8, n. 3, p. 311-331. 1998. http://dx.doi.org/10.1016/ S1053-4822(98)90007-9

SANDRONI, P. Novissímo dicionário de economia. São Paulo: Best Seller, 1999.

SANZ-VALLE, R.; SÁNCHEZ, R. S. Enfoque universalista de recursos humanos: estudio empírico de sus efectos sobre los resultados de la empresa. Revista Europea de Dirección y Economía de la Empresa, v. 11, n. 1, p. 163-182, 2002.

SCHULER, R. S.; JACKSON, S. E. Organizational strategy and organizational level as determinants of human resource management practices. Human Resource Planning, v. 10, n. 3, p. 125-141, 1987.

STAHL, M. J. CEO compensation as a psychological exit barrier: an exploratory test with HMOs. Psychology and Marketing, v. 17, n. 5, p. 387-395, 2000. http://dx.doi. org/10.1002/(SICI)1520-6793(200005)17:5<387::AIDMAR2>3.0.CO;2-H

STAVROU-COSTEA, E. T.; CHARALAMBOUS, C.; SPILIOTIS, S. Human resource management and performance: a neural network analysis. European Journal of Operational Research, v. 181, n. 1, p. 453-467, 2007. http://dx.doi.org/10.1016/j. ejor.2006.06.006

STOREY, D. J. Education, training and development policies and practices in medium-sized companies in the UK: do they really influence firm performance? Omega: The International Journal of Management Science, v. 30, n. 4, p. 249-264, 2002.

SUBRAMONY, M. A meta-analytic investigation of the relationship between HRM bundles and firm performance. Human Resource Management, v. 48, n. 5, p. 745-768. 2009. http://dx.doi.org/10.1002/hrm.20315

TREGASKIS, O. The role of national context and HR strategy in shaping training and development practice in French and UK organizations. Organization Studies, v. 18, n. 5, p. 839-856, 1997. http://dx.doi. org/10.1177/017084069701800507

TZAFRIR, S. S. The relationship between trust, HRM practices and firm performance. International Journal of Human Resource Management, v. 16, n. 9, p. 1600-1622, 2005. http://dx.doi.org/10.1080/09585190500239135

WANG, D. et al. Employment relationships and firm performance: evidence from an emerging economy. Journal of Organizational Behavior, v. 24, n. 5, p. 511-535, 2003. http://dx.doi.org/10.1002/job.213

WEBSTER, E.; WOOD, G. Human resource management practice and institutional constraints: the case of Mozambique. Employee Relations, v. 27 , n. 4-5, p. 369-385, 2005. http://dx.doi. org/10.1108/01425450510605705
WOOD, S. J. Human resource management and performance. International Journal of Management Reviews, v. 1, n. 4, p. 367-413, 1999. http://dx.doi. org/10.1111/1468-2370.00020

WRIGHT, P. M. et al. The relationship between HR practices and firm performance: examining causal order. Personnel Psychology, v. 58, n. 2, p. 409-446, 2005. http://dx.doi.org/10.1111/j.1744-6570.2005.00487.x

WRIGHT, P. M.; SNELL, S. A.; DYER, L. New models of strategic HRM in a global context. International Journal of Human Resource Management, v. 16, n. 6, p. $875-881,2005$. http://dx.doi. org/10.1080/09585190500120814

YOUNDT, M. A. et al. Human resource management, manufacturing strategy, and firm performance. Academy of Management Journal, v. 39, n. 4, p. 836-866, 1996. http://dx.doi.org/10.2307/256714

ZACHARATOS, A.; BARLING, J.; IVERSON, R. D. High-performance work systems and occupational safety. Journal of Applied Psychology, v. 90, n. 1, p. 77-93, 2005. PMid:15641891. http://dx.doi. org/10.1037/0021-9010.90.1.77

\section{Bibliografia analisada}

AGARWALA, T. Innovative human resource practices and organizational commitment: an empirical investigation. International Journal of Human Resource Management, v. 14, n. 2, p. 175-197, 2003. http:// dx.doi.org/10.1080/0958519021000029072

AHMAD, S.; SCHROEDER, R. G. The impact of human resource management practices on operational performance: recognizing country and industry differences. Journal of Operations Management, v. 21, n. 1, p. 19-43, 2003. http://dx.doi.org/10.1016/ S0272-6963(02)00056-6

ALLEN, R. S.; KILMANN, R. H. The role of the reward system for a total quality management based strategy. Journal of Organizational Chance Management, v. 14, n. 2, p. 110-131, 2001. http:// dx.doi.org/10.1108/09534810110388036

APPLEYARD, M. M.; BROWN, C. Employment practices and semiconductor manufacturing performance. Industrial Relations, v. 40, n. 3, p. 436-471, 2001. http://dx.doi.org/10.1111/0019-8676.00219

ARTHUR, J. B. Effects of human resource systems on manufacturing performance and turnover. Academy of Management Journal, v. 37, n. 3, p. 670-687, 1994. http://dx.doi.org/10.2307/256705

AUDEA, T.; TEO, S. T. T.; CRAWFORD, J. HRM professionals and their perceptions of HRM and firm performance in the Philippines. International Journal of Human Resource Management, v. 16, n. 4, p. 532-552, 2005. http://dx.doi.org/10.1080/09585190500051589

BACON, N.; BLYTON, P. High road and low road teamworking: perceptions of management rationales and organizational and human resource outcomes. Human Relations, v. 53, n. 11, p. 1425-1458, 2000.

BAE, J. et al. Human resource strategy and firm performance in Pacific Rim countries. International Journal of 
Human Resource Management, v. 14, n. 8, p. 1308-1332, 2003. http://dx.doi.org/10.1080/0958519032000145774

BAE, J.; LAWLER, J. J. Organizational and HRM strategies in Korea: impact on firm performance in an emerging economy. Academy of Management Journal, v. 43, n. 3, p. 502-517, 2000. http://dx.doi.org/10.2307/1556407

BAMBERGER, P.; BACHARACH, S. B.; DYER, L. Human resources management and organizational effectiveness: high technology entrepreneurial startup firms in Israel. Human Resource Management, v. 28, n. 3, p. 349-366, 1989. http://dx.doi.org/10.1002/hrm.3930280305

BANKER, R. D. et al. Impact of work teams on manufacturing performance: a longitudinal field study. Academy of Management Journal, v. 39, n. 4, p. 867-890, 1996a. http://dx.doi.org/10.2307/256715

BANKER, R. D. et al. Contextual analysis of performance impacts of outcome-based incentive compensation. Academy of Management Journal, v. 39, n. 4, p. 920-948, 1996b. http://dx.doi.org/10.2307/256717

BATT, R. Work organization, technology, and performance in customer service and sales. Industrial and Labor Relations Review, v. 52, n. 4, p. 539-564, 1999. http:// dx.doi.org/10.2307/2525063

BATT, R.; COLVIN, A. J. S.; KEEFE, J. Employee voice, human resource practices, and quit rates: evidence from the telecommunications industry. Industrial and Labor Relations Review, v. 55, n. 4, p. 573-594, 2002. http:// dx.doi.org/10.2307/3270623

BATT, R.; VALCOUR, P. M. Human resources practices as predictors of work-family outcomes and employee turnover. Industrial Relations, v. 42, n. 2, p. 189-220, 2003. http://dx.doi.org/10.1111/1468-232X.00287

BAYO-MORIONES, A.; DE CERIO, J. M. D. Human resource management and operational performance in Spanish manufacturing industry. Pamplona: Departamento de Gestión de Empresas: Universidad Pública de Navarra, 2000.

BAYO-MORIONES, A.; DE CERIO, J. M. D. Employee involvement: its interaction with advanced manufacturing technologies, quality management, and inter-firm collaboration. Human Factors and Ergonomics in Manufacturing, v. 14, n. 2, p. 117-134, 2004. http:// dx.doi.org/10.1002/hfm.10057

BJORKMAN, I.; XIUCHENG, F. Human resource management and the performance of Western firms in China. International Journal of Human Resource Management, v. 13, n. 6, p. 853-864, 2002. http:// dx.doi.org/10.1080/09585190210134246

BLACK, S. E.; LYNCH, L. M. How to compete: the impact of workplace practices and information technology on productivity. Review of Economics and Statistics, v. 83, n. 3, p. 434-445, 2001. http:// dx.doi.org/10.1162/00346530152480081

BLACK, S. E.; LYNCH, L. M. What's driving the new economy? The benefits of workplace innovation. Economic Journal, v. 114, n. 2, p. 97-116, 2004. http://dx.doi.org/10.1111/j.0013-0133.2004.00189.x

BOSELIE, P.; PAAUWE, J.; RICHARDSON, R. Human resource management, institutionalization and organizational performance: a comparison of hospitals, hotels and local government. International
Journal of Human Resource Management, v. 14, n. 8 , p. 1407-1429, 2003. http://dx.doi. org/10.1080/0958519032000145828

BOSELIE, P.; VAN DER WIELE, T. Employee perceptions of HRM and TQM, and the effects on satisfaction and intention to leave. Managing Service Quality, v. 12, n. 3, p. 165-172, 2002. http://dx.doi. org/10.1108/09604520210429231

BOU, J. C. L.; BELTRÁN, I. Total quality management, high-commitment human resource strategy and firm performance: an empirical study. Total Quality Management and Business Excellence, v. 16, n. 1, p. 71-86, 2005. http://dx.doi. org/10.1080/1478336042000309875

BROWN, M. P.; STURMAN, M. C.; SIMMERING, M. J. Compensatory policy and organizational performance: the efficiency, operational, and financial implications of pay levels and pay structure. Academy of Management Journal, v. 46, n. 6, p. 752-762, 2003. http://dx.doi. org/10.2307/30040666

BRYSON, A.; FORTH, J.; KIRBY, S. High-involvement management practices, trade union representation and workplace performance in Britain. Scottish Journal of Political Economy, v. 52, n. 3, p. 451-491, 2005. http://dx.doi.org/10.1111/j.0036-9292.2005.00352.x

BUCK, T. et al. Insider ownership, human resource strategies and performance in a transition economy. Journal of International Business Studies, v. 34, n. 6, p. 530-549, 2003. http://dx.doi.org/10.1057/palgrave.jibs.8400065

BURTON, M. D.; O'REILLY III, C. A. The impact of high commitment values and practices on technology start-ups. In: ANNUAL MEETING OF THE ACADEMY OF MANAGEMENT, 2000, Toronto, Anais...

CHADWICK, C. Examining non-linear relationships between human resource practices and manufacturing performance. Industrial and Labor Relations Review, v. 60, n. 4, p. 499-521, 2007.

CHADWICK, C.; HUNTER, L. W.; WALSTON, S. L. Effects of downsizing practices on the performance of hospitals. Strategic Management Journal, v. 25, n. 5, p. 405-427, 2004. http://dx.doi.org/10.1002/smj.383

CHALLIS, D.; SAMSON, D.; LAWSON, B. Impact of technological, organizational and human resource investments on employee and manufacturing performance: Australian and New Zealand evidence. International Journal of Production Research, v. 43, n. 1, p. 81-107, 2005. http://dx.doi.org/10.1080/00207540412331281935

CHAN, L. L. M.; SHAFFER, M. A.; SNAPE, E. In search of sustained competitive advantage: the impact of organizational culture, competitive strategy and human resource management practices on firm performance. International Journal of Human Resource Management, v. 15, n. 1, p. 17-35, 2004. http://dx.doi. org/10.1080/0958519032000157320

CHANDLER, G. N.; KELLER, C.; LYON, D. W. Unraveling the determinants and consequences of an innovationsupportive organizational culture. Entrepreneurship Theory and Practice, v. 25, n. 1, p. 59-76, 2000.

CHANDLER, G. N.; MCEVOY, G. M. Human resource management, TQM, and firm performance in small and 
medium-size enterprises. Entrepreneurship Theory and Practice, v. 25, n. 1, p. 43-57, 2000.

CHANG, E. Employees' overall perception of HRM effectiveness. Human Relations, v. 58, n. 4, p. 523-544, 2005. http://dx.doi.org/10.1177/0018726705055037

CHANG, W. J. A.; HUANG, T. C. Relationship between strategic human resource management and firm performance: a contingency perspective. International Journal of Manpower, v. 26, n. 5, p. 434-449, 2005. http://dx.doi.org/10.1108/01437720510615125

COLLINS, C. J. Strategic human resource management and knowledge-creation capability: examining the black box between HR and firm performance. 2000. Dissertação (Doctor of Philosophy)-University of Maryland, Maryland, 2000.

COLLINS, C. J.; CLARK, K. D. Strategic human resource practices, top management team social networks, and firm performance: the role of human resource practices in creating organizational competitive advantage. Academy of Management Journal, v. 46, n. 6, p. 740-751, 2003. http://dx.doi.org/10.2307/30040665

COLLINS, C. J.; SMITH, K. G. Knowledge exchange and combination: the role of human resource practices in the performance of high-technology firms. Academy of Management Journal, v. 49, n. 3, p. 544-560, 2006. http://dx.doi.org/10.5465/AMJ.2006.21794671

CONWAY, E. Relating career stage to attitudes towards HR practices and commitment: evidence of interaction effects? European Journal of Work and Organizational Psychology, v. 13, n. 4, p. 417-446, 2004. http://dx.doi. org/10.1080/13594320444000155

COSH, A.; HUGHES, A.; WEEKS, M. The relationship between training and employment growth in small and medium-sized enterprises. Cambridge: University of Cambridge, 2000. PMCid:PMC1117376

COWELL, N. M. The use of workplace innovations in a developing economy: the case of Jamaica. 2000. Tese (Doctor of Philosophy)-University of Toronto, Toronto, 2000.

CUNHA, R. C. E. et al. Market forces, strategic management, HRM practices and organizational performance: a model based in a European sample. Rochester: SSRN, 2003. (FEUNL Working Paper, n. 415).

DAS, A. et al. A contingent view of quality management: the impact of international competition on quality. Decision Sciences, v. 31, n. 3, p. 649-690, 2000.

DATTA, D. K.; GUTHRIE, J. P.; WRIGHT, P. M. Human resource management and labor productivity: Does industry matter? Academy of Management Journal, v. 48, n. 1, p. 135-145, 2005. http://dx.doi.org/10.5465/ AMJ.2005.15993158

DE PABLOS, P. O. Capital intelectual, gestión del conocimiento y sistemas de gestión de recursos humanos: influencia sobre los resultados organizativos en la industria manufacturera española. 2001. Tese (Doctorado en Administración y Dirección de Empresas)-Universidad de Oviedo, Oviedo, 2001. PMCid:PMC311117

DECKOP, J. R. et al. The effect of human resource management practices on the job retention of former welfare clients. Human Resource Management, v. 45, n. 4, p. 539-559, 2006. http://dx.doi.org/10.1002/ hrm.20131

DELERY, J. E. et al. Unionization, compensation, and voice effects on quits and retention. Industrial Relations, v. 39, n. 4, p. 625-645, 2000. http://dx.doi. org/10.1111/0019-8676.00186

DELERY, J. E.; DOTY, D. H. Modes of theorizing in strategic human resource management: tests of universalistic, contingency, and configurational performance predictions. Academy of Management Journal, v. 39, n. 4, p. 802-835, 1996. http://dx.doi.org/10.2307/256713

DEN HARTOG, D. N.; VERBURG, R. M. High performance work systems, organisational culture and firm effectiveness. Human Resource Management Journal, v. 14, n. 1, p. 55-78, 2004. http://dx.doi. org/10.1111/j.1748-8583.2004.tb00112.x

FERRATT, T. W. et al. IT human resource management configurations and IT turnover: theoretical synthesis and empirical analysis. Information Systems Research, v. 16, n. 3, p. 237-255, 2005. http://dx.doi.org/10.1287/ isre. 1050.0057

FEY, C. F.; BJORKMAN, I.; PAVLOVSKAYA, A. The effect of human resource management practices on firm performance in Russia. International Journal of Human Resource Management, v. 11, n. 1, p. 1-18, 2000. http://dx.doi.org/10.1080/095851900339963

FURUYA, N. et al. The effects of HR policies and repatriate self-adjustment on global competency transfer. Asia Pacific Journal of Human Resources, v. 45, n. 1, p. 6-23, 2007. http://dx.doi.org/10.1177/1038411107073595

GERHART, B.; MILKOVICH, G. T. Organizational differences in managerial compensation and financial performance. Academy of Management Journal, v. 33, n. 4, p. 663-691, 1990. http://dx.doi.org/10.2307/256286

GODARD, J. Beyond the high-performance paradigm? An analysis of variation in Canadian managerial perceptions of reform programme effectiveness. British Journal of Industrial Relations, v. 39, n. 1, p. 25-52, $2001 \mathrm{a}$. http://dx.doi.org/10.1111/1467-8543.00188

GODARD, J. High performance and the transformation of work? The implications of alternative work practices for the experience and outcomes of work. Industrial and Labor Relations Review, v. 54, n. 4, p. 776-805, 2001b. http://dx.doi.org/10.2307/2696112

GÓMEZ-MEJÍA, L. R. The role of human resources strategy in export performance: a longitudinal study. Strategic Management Journal, v. 9, n. 5, p. 493-505, 1988. http://dx.doi.org/10.1002/smj.4250090508

GONZÁLEZ, J. V.; GARAZO, T. G. Structural relationships between organizational service orientation, contact employee job satisfaction and citizenship behavior. International Journal of Service Industry Management, v. 17, n. 1, p. 23-50, 2006. http://dx.doi. org/10.1108/09564230610651561

GOULD WILLIAMS, J. The importance of HR practices and workplace trust in achieving superior performance: a study of public-sector organizations. International Journal of Human Resource Management, v. 14, n. 1, p. 28-54, 2003. http://dx.doi.org/10.1080/09585190210158501 GREER, C. R.; IRELAND, T. C.; WINGENDER, J. R. Contrarian human resource investments and financial 
performance after economic downturns. Journal of Business Research, v. 52, n. 3, p. 249-261, 2001. http:// dx.doi.org/10.1016/S0148-2963(99)00108-3

GUERRERO, S.; BARRAUD-DIDIER, V. Highinvolvement practices and performance of French firms. International Journal of Human Resource Management, v. 15, n. 8, p. 1408-1423, 2004. http:// dx.doi.org/10.1080/0958519042000258002

GUEST, D. E. et al. Human resource management and corporate performance in the UK. British Journal of Industrial Relations, v. 41, n. 2, p. 291-314, 2003. http://dx.doi.org/10.1111/1467-8543.00273

GUTHRIE, J. P. High-involvement work practices, turnover, and productivity: evidence from New Zealand.Academy of Management Journal, v. 44, n. 1, p. 180-190, 2001. http://dx.doi.org/10.2307/3069345

HANSSON, B. Company-based determinants of training and the impact of training on company performance: results from an international HRM survey. Personnel Review, v. 36, n. 2, p. 311-331, 2007. http://dx.doi. org/10.1108/00483480710726163

HAREL, G. H.; TZAFRIR, S. S. The effect of human resource management practices on the perceptions of organizational and market performance of the firm. Human Resource Management, v. 38, n. 3, p. 185-200, 1999. http://dx.doi.org/10.1002/(SICI)1099050X(199923)38:3<185::AID-HRM2>3.0.CO;2-Y

HATCH, N. W.; DYER, J. H. Human capital and learning as a source of sustainable competitive advantage. Strategic Management Journal, v. 25, n. 12, p. 1155-1178, 2004. http://dx.doi.org/10.1002/smj.421

HAYTON, J. C. Strategic human capital management in SMEs: an empirical study of entrepreneurial performance. Human Resource Management, v. 42, n. 4, p. 375-391, 2003. http://dx.doi.org/10.1002/hrm.10096

HOON, L. S. A managerial perspective of the objectives of HRM practices in Singapore: an exploratory study. Singapore Management Review, v. 22, n. 1, p. 65-82, 2000.

HUANG, T. C. Are the human resource practices of effective firms distinctly different from those of poorly performing ones? Evidence from Taiwanese enterprises. International Journal of Human Resource Management, v. 11, n. 2, p. 436-451, 2000. http:// dx.doi.org/10.1080/095851900339945

HUANG, T. C. Succession management systems and human resource outcomes. International Journal of Manpower, v. 22, n. 7-8, p. 736-747, 2001. http:// dx.doi.org/10.1108/EUM0000000006508

HUNTER, L. W.; HITT, L. M. What makes a highperformance workplace? Evidence from retail bank branches. Pennsylvania: University of Pennsylvania, 2000.

HUSELID, M. A. The impact of human resource management practices on turnover, productivity, and corporate financial performance. Academy of Management Journal, v. 38, n. 3, p. 635-672, 1995. http://dx.doi.org/10.2307/256741

HUSELID, M. A.; BECKER, B. E. The strategic impact of high performance work systems. New Brunswick: Human Resource Planning Society: Society for Human Resource Management Foundation: School of Management and
Labor Relations at Rutgers University: SUNY-Buffalo School of Management, 1995. (Relatório de Pesquisa).

HUSELID, M. A.; BECKER, B. E. The impact high performance work systems, implementation effectiveness, and alignment with strategy on shareholder wealth. In: ACADEMY OF MANAGEMENT PROCEEDINGS, 1997, Boston. Anais...

HUSELID, M. A.; JACKSON, S. E.; SCHULER, R. S. Technical and strategic human resource management effectiveness as determinants of firm performance. Academy of Management Journal, v. 40, n. 1, p. 171-188, 1997. http://dx.doi.org/10.2307/257025

JAW, B. S.; LIU, W. Promoting organizational learning and self-renewal in Taiwanese companies: the role of HRM. Human Resource Management, v. 42, n. 3, p. 223-241, 2003. http://dx.doi.org/10.1002/hrm.10082

JAYARAM, J.; VICKERY, S. K. Supply-based strategies, human resource initiatives, procurement leadtime, and firm performance. International Journal of Purchasing and Materials Management, v. 34, n. 1, p. 12-23, 1989.

JEHN, K. A.; BEZRUKOVA, K. A field study of group diversity, workgroup context, and performance. Journal of Organizational Behavior, v. 25, n. 6, p. 703-729, 2004. http://dx.doi.org/10.1002/job.257

JEREZ-GÓMEZ, P.; CÉSPEDES-LORENTE, J.; VALLECABRERA, R. Organizational learning and compensation strategies: evidence from the Spanish chemical industry. Human Resource Management, v. 44, n. 3, p. 279-299, 2005. http://dx.doi.org/10.1002/hrm.20071

JIMÉNEZ-JIMÉNEZ, D.; SANZ-VALLE, R. Innovation and human resource management fit: an empirical study. International Journal of Manpower, v. 26, n. 4, p. 364-381, 2005. http://dx.doi. org/10.1108/01437720510609555

KAMAN, V. et al. Bureaucratic and high commitment human resource practices in small service firms. Human Resource Planning, v. 24, n. 1, p. 33-44, 2001.

KATOU, A. A.; BUDHWAR, P. S. Human resource management systems and organizational performance: a test of a mediating model in the Greek manufacturing context. International Journal of Human Resource Management, v. 17, n. 7, p. 1223-1253, 2006. http:// dx.doi.org/10.1080/09585190600756525

KATZ, H. C.; KOCHAN, T. A.; WEBER, M. R. Assessing the effects of industrial relations systems and efforts to improve the quality of working life on organizational effectiveness. Academy of Management Journal, v. 28, n. 3, p. 509-526, 1985. http://dx.doi.org/10.2307/256111

KAYA, N. The impact of human resource management practices and corporate entrepreneurship on firm performance: evidence from Turkish firms. International Journal of Human Resource Management, v. 17, n. 12, p. 2074-2090, 2006. http://dx.doi. org/10.1080/09585190601000204

KHATRI, N. Managing human resource for competitive advantage: a study of companies in Singapore. International Journal of Human Resource Management, v. 11, n. 2, p. 336-365, 2000. http:// dx.doi.org/10.1080/095851900339909

KHILJI, S. E.; WANG, X. 'Intended' and 'implemented' HRM: the missing linchpin in strategic human resource 
management research. International Journal of Human Resource Management, v. 17, n. 7, p. 1171-1189, 2006. http://dx.doi.org/10.1080/09585190600756384

KIM, D. O. The benefits and costs of employee suggestions under gainsharing. Industrial and Labor Relations Review, v. 58, n. 4, p. 631-652, 2005.

KIM, Y. M.; PARK, K. S. Multiskilling and firm performance. Seoul Journal of Economics, v. 16, n. 4, p. 387-422, 2003.

KING-KAUANUI, S.; NGOC, S. D.; ASHLEY-COTLEUR,

C. Impact of human resource management: SME performance in Vietnam. Journal of Developmental Entrepreneurship, v. 11, n. 1, p. 79-95, 2006. http:// dx.doi.org/10.1142/S1084946706000271

KINTANA, M. L.; ALONSO, A. U.; OLAVERRI, M. C. G. High performance work systems and firm's operational performance: the moderating role of technology. International Journal of Human Resource Management, v. 17, n. 1, p. 70-85, 2006. http://dx.doi. org/10.1080/09585190500366466

KONRAD, A. M.; MANGEL, R. The impact of work-life programs on firm productivity. Strategic Management Journal, v. 21, n. 12, p. 1225-1237, 2000. http://dx.doi. org/10.1002/1097-0266(200012)21:12<1225::AIDSMJ135>3.0.CO;2-3

LAM, L. W.; WHITE, L. P. Human resource orientation and corporate performance. Human Resource Development Quarterly, v. 9, n. 4, p. 351-364, 1998. http://dx.doi. org/10.1002/hrdq.3920090406

LEE, J.; MILLER, D. People matter: commitment to employees, strategy and performance in Korea firms. Strategic Management Journal, v. 20, n. 6, p. 579-593, 1999. http://dx.doi.org/10.1002/(SICI)10970266(199906)20:6<579::AID-SMJ37>3.0.CO;2-C

LEE, S. H.; PHAN, P. H.; CHAN, E. The impact of HR configuration on firm performance in Singapore: a resource-based explanation. International Journal of Human Resource Management, v. 16, n. 9, p. 1740-1758, 2005. http://dx.doi.org/10.1080/09585190500239465

LEPAK, D. P.; SNELL, S. A. Examining the human resource architecture: the relationships among human capital, employment, and human resource configurations. Journal of Management, v. 28, n. 4, p. 517-543, 2002. http:// dx.doi.org/10.1016/S0149-2063(02)00142-3

LEVENSON, A. R.; VAN DER STEDE, W. A.; COHEN, S. G. Measuring the relationship between managerial competencies and performance. Journal of Management, v. 32, n. 3, p. 360-380, 2006. http:// dx.doi.org/10.1177/0149206305280789

LI, J. Strategic human resource management and MNEs' performance in China. International Journal of Human Resource Management, v. 14, n. 2, p. 157-173, 2003. http://dx.doi.org/10.1080/0958519021000029063

LI, Y.; ZHAO, Y.; LIU, Y. The relationship between HRM, technology innovation and performance in China. International Journal of Manpower, v. 27, n. 7, p. 679-697, 2006. http://dx.doi. org/10.1108/01437720610708284

LIAO, Y. S. Business strategy and performance: the role of human resource management control. Personnel
Review, v. 34, n. 3, p. 294-309, 2005. http://dx.doi. org/10.1108/00483480510591444

LIAO, Y. S. The effect of fit between organizational life cycle and human resource management control on firm performance. Journal of American Academy of Business, v. 8, n. 1, p. 192-196, 2006 a.

LIAO, Y. S. Human resource management control system and firm performance: a contingency model of corporate control. International Journal of Human Resource Management, v. 17, n. 4, p. 716-733, 2006b. http:// dx.doi.org/10.1080/09585190600581691

LIOUVILLE, J.; BAYAD, M. Human resource management and performances: proposition and test of a causal model. Human Systems Management, v. 17, n. 3, p. 183-192, 1998.

LITZ, R. A.; STEWART, A. C. Research Note: trade name franchise membership as a human resource management strategy. Does buying group training deliver 'true value' for small retailers? Entrepreneurship Theory and Practice, v. 25, n. 1, p. 125, 2000.

LÓPEZ, S. P.; PEÓN, J. M. M.; ORDÁS, C. J. V. Prácticas de recursos humanos de alto rendimiento y aprendizaje organizativo: efectos sobre los resultados empresariales. Revista Icade, n. 65, p. 75-97, 2005.

LUCARELLI, C. M. Developing competencies and capabilities through human capital development. 2004 Tese (Doctor of Philosophy)-Rensselaer Polytechnic Institute, New York, 2004. PMCid:PMC1304290

LUTHANS, F.; PETERSON, S. J. 360-degree feedback with systematic coaching: Empirical analysis suggests a winning combination. Human Resource Management, v. 42, n. 3, p. 243-256, 2003. http://dx.doi.org/10.1002/ hrm. 10083

MABEY, C.; GOODERHAM, P. N. The impact of management development on perceptions of organizational performance in European firms. European Management Review, v. 2, n. 2, p. 131-142, 2005. http://dx.doi.org/10.1057/palgrave.emr.1500032

MABEY, C.; RAMIREZ, M. Does management development improve organizational productivity? A six-country analysis of European firms. International Journal of Human Resource Management, v. 16, n. 7, p. 1067-1082, 2005. http://dx.doi.org/10.1080/09585190500143931

MACDUFFIE, J. P. Human resource bundles and manufacturing performance: organizational logic and flexible production systems in the world auto industry. Industrial and Labor Relations Review, v. 48, n. 2, p. 197-221, 1995. http://dx.doi.org/10.2307/2524483

MACKY, K.; BOXALL, P. The relationship between 'high-performance work practices' and employee attitudes: an investigation of additive and interaction effects. International Journal of Human Resource Management, v. 18, n. 4, p. 537-567, 2007. http:// dx.doi.org/10.1080/09585190601178745

MACKY, K.; BOXALL, P. High-involvement work processes, work intensification and employee well-being: a study of New Zealand worker experiences. Asia Pacific Journal of Human Resources, v. 46, n. 1, p. 38-55, 2008. http://dx.doi.org/10.1177/1038411107086542

MAGNAN, M. L.; ST-ONGE, S. Bank performance and executive compensation: a managerial discretion 
perspective. Strategic Management Journal, v. 18, n. 7, p. 573-581, 1997. http://dx.doi.org/10.1002/(SICI)10970266(199708)18:7<573::AID-SMJ900>3.0.CO;2-K

MAK, S. K. M.; AKHTAR, S. Human resource management practices, strategic orientations, and company performance: a correlation study of publicly listed companies. Journal of American Academy of Business, v. 2, n. 2, p. 510-515, 2003.

MARTELL, K.; CARROLL, S. J. Which executive human resource management practices for the top management team are associated with higher firm performance? Human Resource Management, v. 34, n. 4, p. 497-512, 1995. http://dx.doi.org/10.1002/hrm.3930340403

MCMILLAN-CAPEHART, A. Cultural diversity's impact on firm performance: the moderating influence of diversity initiatives and socialization tactics. 2003. Tese (Doctor of Business Administration)-Louisiana Tech University, Louisiana, 2003.

MEYER, J. P.; SMITH, C. A. HRM practices and organizational commitment: test of a mediation model. Canadian Journal of Administrative Sciences, v. 17, n. 4, p. 319-331, 2000. http://dx.doi. org/10.1111/j.1936-4490.2000.tb00231.x

MONTEMAYOR, E. F. Congruence between pay policy and competitive strategy in high-performing firms. Journal of Management, v. 22, n. 6, p. 889-908, 1996. http:// dx.doi.org/10.1177/014920639602200605

NEAL, A.; WEST, M. A.; PATTERSON, M. G. Do organizational climate and competitive strategy moderate the relationship between human resource management and productivity? Journal of Management, v. 31, n. 4, p. 492-512, 2005. http:// dx.doi.org/10.1177/0149206304272188

NGO, H. Y. et al. Human resource practices and firm performance of multinational corporations: influences of country origin. International Journal of Human Resource Management, v. 9, n. 4, p. 632-652, 1998. http://dx.doi.org/10.1080/095851998340937

NGUYEN, T. V.; BRYANT, S. E. A study of the formality of human resource management practices in small and medium-size enterprises in Vietnam. International Small Business Journal, v. 22, n. 6, p. 595-618, 2004. http://dx.doi.org/10.1177/0266242604047412

NOBLE, D. S. Human resource management strategy: the dual pursuit of employee involvement and workforce adaptability. 2000. Tese (Doctor of Philosophy)-Wayne State University, Detroit, 2000.

NOWICKI, M. D. Exploring the effect of a climate for service on the SHRM-firm performance relationship. 2001. Tese (Doctor of Philosophy)-University of Colorado, Boulder, Colorado, 2001.

OGUNRINDE, R. O. Strategic human resource planning and organizational effectiveness: an empirical analysis and recommendations. 2001. Tese (Doctor of Public Administration)-Nova South-Eastern University, Florida, 2001.

PARK, H. J. et al. The effect of human resource management practices on Japanese MNC subsidiary performance: a partial mediating model. International Journal of Human Resource Management, v. 14, n. 8, p. 1391-1406, 2003. http://dx.doi.org/10.1080/0958519032000145819
PATTERSON, M. G. et al. Impact of people management practices on business performance. London: Institute of Personnel and Development, 1997. 39 p. (Issues in People Management).

PATTERSON, M. G.; WEST, M. A.; WALL, T. D. Integrated manufacturing, empowerment, and company performance. Journal of Organizational Behavior, v. 25 , n. 6, p. 641-665, 2004. http://dx.doi.org/10.1002/ job. 261

PAUL, A. K.; ANANTHARAMAN, R. N. Impact of people management practices on organizational performance: analysis of a causal model. International Journal of Human Resource Management, v. 14, n. 7, p. 1246-1266, 2003. http://dx.doi.org/10.1080/0958519032000145648

PERRY SMITH, J. E.; BLUM, T. C. Work-family human resource bundles and perceived organizational performance. Academy of Management Journal, v. 43, n. 6, p. 1107-1117, 2000. http://dx.doi. org/10.2307/1556339

PFAU, B. N.; COHEN, S. A. Aligning human capital practices and employee behavior with shareholder value. Consulting Psychology Journal: Practice and Research, v. 55, n. 3, p. 169-178, 2003. http://dx.doi. org/10.1037/1061-4087.55.3.169

PIEKKOLA, H. Performance-related pay and firm performance in Finland. International Journal of Manpower, v. 6, n. 7-8, p. 619-635, 2005. http://dx.doi. org/10.1108/01437720510628103

PREUSS, G. A. High performance works systems and organizational outcomes: the mediating role of information quality. Industrial and Labor Relations Review, v. 56, n. 4, p. 590-605, 2003. http://dx.doi. org/10.2307/3590958

RAMSAY, H.; SCHOLARIOS, D.; HARLEY, B. Employees and high-performance work systems: testing inside the black box. British Journal of Industrial Relations, v. 38, n. 4, p. 501-531, 2000. http://dx.doi. org/10.1111/1467-8543.00178

RAUCH, A.; FRESE, M.; UTSCH, A. Effects of human capital and long-term human resources development and utilization on employment growth of small-scale businesses: a causal analysis. Entrepreneurship Theory and Practice, v. 29, n. 6, p. 681-698, 2005.

RICHARD, O. C.; JOHNSON, N. B. Strategic human resource management effectiveness and firm performance. International Journal of Human Resource Management, v. 12, n. 2, p. 299-310, 2001. http:// dx.doi.org/10.1080/09585190121674

RICHARD, O. C.; JOHNSON, N. B. High performance work practices and human resource management effectiveness: substitutes or complements? Journal of Business Strategies, v. 21, n. 2, p. 133-148, 2004.

ROCA-PUIG, V.; TENA, A. B. E.; BOU, J. C. L. A methodological approach to studying the fit between commitment management and competitive strategy. Management Research, v. 2, n. 2, p. 161-174, 2004.

RODWELL, J. J.; TEO, S. T. T. Strategic HRM in for-profit and non-profit organizations in a knowledge-intensive industry: the same issues predict performance for both types of organization. Public Management 
Review, v. 6, n. 3, p. 311-331, 2004. http://dx.doi. org/10.1080/1471903042000256510

ROGG, K. L. et al. Human resource practices, organizational climate and customer satisfaction. Journal of Management, v. 27, n. 4, p. 431-449, 2001. http:// dx.doi.org/10.1177/014920630102700403

RONDEAU, K. V.; WAGAR, T. H. Impact of human resource management practices on nursing home performance. Health Services Management Research, v. 14, n. 3, p. 192-202, 2001. PMid:11507813. http:// dx.doi.org/10.1258/0951484011912690

RUSSELL, J. S.; TERBORG, J. R.; POWERS, M. L. Organizational performance and organizational level training and support. Personnel Psychology, v. 38, n. 4 , p. 849-863, 1985. http://dx.doi. org/10.1111/j.1744-6570.1985.tb00570.x

SAÁ-PÉREZ, P. D. El sistema de recursos humanos como factor determinante de la competitividad de las Cajas de Ahorros españolas: una aplicación de la teoría de la empresa basada en los recursos. 1999. Tese (Doctorado en Ciencias Econômicas y Empresariales)Universidad Las Palmas de Gran Canaria, Las Palmas de Gran Canaria, 1999.

SANZ-VALLE, R.; SÁNCHEZ, R. S. Enfoque universalista de recursos humanos: estudio empírico de sus efectos sobre los resultados de la empresa. Revista Europea de Dirección y Economía de la Empresa, v. 11, n. 1, p. 163-182, 2002.

SATTLER, L.; SOHONI, V. Participative management: an empirical study of the semiconductor manufacturing industry. 1999. (Manuscrito não publicado).

SELS, L. et al. Unravelling the HRM-performance link: value-creating and cost-increasing effects of small business HRM. Journal of Management Studies, v. 43, n. 2, p. 319-342, 2006. http://dx.doi. org/10.1111/j.1467-6486.2006.00592.x

SHAW, J. D. et al. An organization-level analysis of voluntary and involuntary turnover. Academy of Management Journal, v. 41, n. 5, p. 511-525, 1998. http://dx.doi. org/10.2307/256939

SHIH, H. A.; CHIANG, Y. H. Strategy alignment between HRM, KM, and corporate development. International Journal of Manpower, v. 26, n. 6, p. 582-603, 2005. http://dx.doi.org/10.1108/01437720510625476

SHIH, H. A.; CHIANG, Y. H.; HSU, C. C. Can high performance work systems really lead to better performance? International Journal of Manpower, v. 27, n. 8 , p. 741-763, 2006. http://dx.doi. org/10.1108/01437720610713530

SHIPTON, H. et al. Managing people to promote innovation. Creativity and Innovation Management, v. 14, n. 2 , p. 118-128, 2005. http://dx.doi. org/10.1111/j.1467-8691.2005.00332.x

SHIPTON, H. et al. HRM as a predictor of innovation. Human Resource Management Journal, v. 16, n. 1, p. 3-27, 2006. http://dx.doi. org/10.1111/j.1748-8583.2006.00002.x

SIDDIQUE, C. M. Job analysis: a strategic human resource management practice. International Journal of Human Resource Management, v. 15, n. 1, p. 219-244, 2004. http://dx.doi.org/10.1080/0958519032000157438
SINGH, K. Strategic HR orientation and firm performance in India. International Journal of Human Resource Management, v. 14, n. 4, p. 530-543, 2003. http:// dx.doi.org/10.1080/0958519032000057574

SKAGGS, B. C.; YOUNDT, M. A. Strategic positioning, human capital, and performance in service organizations: a customer interaction approach. Strategic Management Journal, v. 25, n. 1, p. 85-99, 2004. http://dx.doi. org/10.1002/smj.365

SNELL, S. A.; YOUNDT, M. A. Human resource management and firm performance: testing a contingency model of executive controls. Journal of Management, v. 21, n. 4, p. 711-737, 1995. http:// dx.doi.org/10.1177/014920639502100407

SPENCER, D. G. Employee voice and employee retention. Academy of Management Journal, v. 29, n. 3, p. 488-502, 1986. PMid:10278801. http://dx.doi. org/10.2307/256220

STAVROU-COSTEA, E. T. Flexible work bundles and organizational competitiveness: a cross-national study of the European work context. Journal of Organizational Behavior, v. 26, n. 8, p. 923-947, 2005. http://dx.doi. org/10.1002/job.356

STEVENS, M. J. et al. HR factors affecting repatriate job satisfaction and job attachment for Japanese managers. International Journal of Human Resource Management, v. 17, n. 5, p. 831-841, 2006. http:// dx.doi.org/10.1080/09585190600640844

SUN, L. Y.; ARYEE, S.; LAW, K. S. High-performance human resource practices, citizenship behavior, and organizational performance: a relational perspective. Academy of Management Journal, v. 50, n. 3, p. 558-577, 2007. http://dx.doi.org/10.5465/ AMJ.2007.25525821

TAKEUCHI, N.; WAKABAYASHI, M.; CHEN, Z. The strategic HRM configuration for competitive advantage: evidence from Japanese firms in China and Taiwan. Asia Pacific Journal of Management, v. 20, n. 4, p. 447-480, 2003. http://dx.doi.org/10.1023/A:1026386925473

TAKEUCHI, R. et al. An empirical examination of the mechanisms mediating between high-performance work systems and the performance of Japanese organizations. Journal of Applied Psychology, v. 92, n. 4, p. 1069-1083, 2007. PMid:17638466. http://dx.doi. org/10.1037/0021-9010.92.4.1069

TARI, J. J.; MOLINA, J. F.; CASTEJÓN, J. L. The relationship between quality management practices and their effects on quality outcomes. European Journal of Operational Research, v. 183, n. 2, p. 483-501, 2007. http://dx.doi.org/10.1016/j.ejor.2006.10.016

TEO, C.; WATERS, L. The role of human resource practices in reducing occupational stress and strain. International Journal of Stress Management, v. 9, n. 3, p. 207-226, 2002. http://dx.doi.org/10.1023/A: 1015575910080

TERPSTRA, D. E.; ROZELL, E. J. The relationship of staffing practices to organizational level measures of performance. Personnel Psychology, v. 46, n. 1, p. 27-48, 1993. http://dx.doi.org/10.1111/j.1744-6570.1993. tb00866.x

TESSEMA, M. T.; SOETERS, J. L. Challenges and prospects of HRM in developing countries: testing 
the HRM-performance link in the Eritrean civil service. International Journal of Human Resource Management, v. 17, n. 1, p. 86-105, 2006. http://dx.doi. org/10.1080/09585190500366532

THANG, L. C.; QUANG, T. Antecedents and consequences of dimensions of human resource management practices in Vietnam. International Journal of Human Resource Management, v. 16, n. 10, p. 1830-1846, 2005. http:// dx.doi.org/10.1080/09585190500298420

THOMPSON, M.; HERON, P. Management capability and high performance work organization. International Journal of Human Resource Management, v. 16, n. 6, p. 1029-1048, 2005. http://dx.doi. org/10.1080/09585190500120806

TSAUR, S. H.; LIN, Y. C. Promoting service quality in tourist hotels: the role of HRM practices and service behavior. Tourism Management, v. 25, n. 4, p. 471-481, 2004. http://dx.doi.org/10.1016/S0261-5177(03)00117-1

TZAFRIR, S. S. et al. The consequences of emerging HRM practices for employees' trust in their managers. Personnel Review, v .33, n. 5-6, p. 628-647, 2004.

VANDENBERG, R. J.; RICHARDSON, H. A.; EASTMAN, L. J. The impact of high involvement work processes on organizational effectiveness: a second-order latent variable approach. Group \& Organization Management, v. 24, n. 3, p. 300-339, 1999. http:// dx.doi.org/10.1177/1059601199243004

VARMA, A. et al. High performance work systems: exciting discovery or passing fad? Human Resource Planning, v. 22, n. 1, p. 26-37, 1999.

VERANO-TACORONTE, D. La influencia de la retribución variable en el rendimiento de la empresa: una aplicación empírica al diseño de la retribución para la fuerza de ventas. 2002. Tese (Doctorado en Economía de las Organizaciones y Dirección Estratégica)-Universidad Las Palmas de Gran Canaria, Las Palmas de Gran Canaria, España, 2002.

VERBURG, R. M.; DEN HARTOG, D. N.; KOOPMAN, P. L. Configurations of human resource management practices: a model and test of internal fit. International Journal of Human Resource Management, v. 18, n. 2, p. 184-208, 2007. http://dx.doi.org/10.1080/09585190601102349

WAN, D.; KOK, V.; ONG, C. H. Strategic human resource management and organizational performance in Singapore. Compensation and Benefits Review, v. 34, n. 4, p. 33-42, 2002. http://dx.doi. org/10.1177/0886368702034004006

WANG, D. et al. Employment relationships and firm performance: evidence from an emerging economy. Journal of Organizational Behavior, v. 24, n. 5, p. 511-535, 2003. http://dx.doi.org/10.1002/job.213

WAY, S. A. High performance work systems and intermediate indicators of firm performance within the US small business sector. Journal of Management, v. 28, n. 6, p. 765-785, 2002. http://dx.doi.org/10.1016/ S0149-2063(02)00191-5

WELBOURNE, T. M.; ANDREWS, A. O. Predicting the performance of initial public offerings: should human resource management be in the equation? Academy of Management Journal, v. 39, n. 4, p. 891-919, 1996. http://dx.doi.org/10.2307/256716

WEST, M. A. et al. The link between the management of employees and patient mortality in acute hospitals. International Journal of Human Resource Management, v. 13, n. 8, p. 1299-1310, 2002. http:// dx.doi.org/10.1080/09585190210156521

WHITENER, E. M. Do "high commitment" human resource practices affect employee commitment? A cross-level analysis using hierarchical linear modelling. Journal of Management, v. 27, n. 5, p. 515-535, 2001.

WILLIAMSON, I. O. Interorganizational ties, institutional pressures and organizational hiring patterns. 2000. Tese (Doctor of Philosophy)-The University of North Carolina at Chapel Hill, Chapel Hill, 2000.

WOOD, S. J.; MENEZES, L. D. High commitment management in the UK: evidence from the workplace industrial relations survey, and employers' manpower and skills practices survey. Human Relations, v. 51, n. 4, p. 485-515, 1998. http://dx.doi. org/10.1177/001872679805100403

WRIGHT, P. M. et al. The role of human resource practices in petro-chemical refinery performance. International Journal of Human Resource Management, v. 10, n. 4, p. 551-571, 1999. http:// dx.doi.org/10.1080/095851999340260

WRIGHT, P. M. et al. The relationship between HR practices and firm performance: examining causal order. Personnel Psychology, v. 58, n. 2, p. 409-446, 2005. http://dx.doi.org/10.1111/j.1744-6570.2005.00487.x

YOUNDT, M. A.; SNELL, S. A. Human resource configurations, intellectual capital, and organizational performance. Journal of Managerial Issues, v. 16, n. 3, p. 337-360, 2004.

ZATZICK, C. D.; IVERSON, R. D. High-involvement management and workforce reduction: competitive advantage or disadvantage? Academy of Management Journal, v. 49, n. 5, p. 999-1015, 2006. http://dx.doi. org/10.5465/AMJ.2006.22798180

ZHU, W.; CHEW, I. K. H.; SPANGLER, W. D. CEO transformational leadership and organizational outcomes: the mediating role of human-capital-enhancing human resource management. Leadership Quarterly, v. 16, n. 1, p. 39-52, 2005. http://dx.doi.org/10.1016/j. leaqua.2004.06.001 